\title{
Article \\ Wavelet Packet Transform Modulus-Based Feature Detection of Stochastic Power Quality Disturbance Signals
}

\author{
Sangho Choe *(D) and Jeonghwa Yoo
}

Citation: Choe, S.; Yoo, J. Wavelet Packet Transform Modulus-Based Feature Detection of Stochastic Power Quality Disturbance Signals. Appl. Sci. 2021, 11, 2825. https://doi.org/ 10.3390/app11062825

Academic Editor: Alfredo Testa

Received: 8 February 2021

Accepted: 17 March 2021

Published: 22 March 2021

Publisher's Note: MDPI stays neutral with regard to jurisdictional claims in published maps and institutional affiliations.

Copyright: (c) 2021 by the authors. Licensee MDPI, Basel, Switzerland. This article is an open access article distributed under the terms and conditions of the Creative Commons Attribution (CC BY) license (https:// creativecommons.org/licenses/by/ $4.0 /)$.
Department of Information, Communications and Electronics Engineering, The Catholic University of Korea, Bucheon-si 14662, Korea; mundade@hanmail.net

* Correspondence: schoe@catholic.ac.kr; Tel.: +82-10-2576-8674

Featured Application: Smart Grid; Power Quality Monitoring Systems; Supervisory Control and Data Acquisition; Advanced Metering Infrastructure.

Abstract: Wavelet transform modulus (WTM) has been used to detect or localize transient signal discontinuities. A numerical analysis indicated that these power quality disturbance (PQD) events are extremely sensitive to the random phase offset due to shift-variant wavelet or wavelet packet characteristics, which have not been comprehensively discussed yet. In this paper, we define wavelet packet transform modulus (WPTM) and present a WPTM-based PQD feature detection that is robust to severe power signal channels including random phase offset and low signal-to-noise ratio $(\leq 25 \mathrm{~dB})$. The presented WPTM-based detection that exhibits an exponentially increased degrees of freedom $(D o F)$ and has better correlation properties than existing WTM-based detection of a limited DoF (two or three). We then use a standard median filter to efficiently remove impulsive noise and add a threshold modification step to reduce the false edge detection rate under random phase offset conditions while maintaining a reasonable detection rate. The proposed scheme uses the majority voting-based indirect correlation or root-mean-square metric between wavelet packet coefficients, rather than the conventional wavelet denoising or correlation metric. For a reliable numerical analysis, the proposed scheme uses both double- and single-edge-based detection measures, and the results verify its superiority over the conventional wavelet-based, wavelet-correlation-based, or non-wavelet-based schemes.

Keywords: adaptive denoising; detection; impulsive noise; phase offset; power quality disturbance (PQD); smart grid; wavelet packet transform (WPT)

\section{Introduction \\ 1.1. Introduction}

Nowadays, owing to the increased use of high-precision equipment, devices, and facilities, power quality disturbance (PQD) issues in a power grid have become a huge concern. A single PQD event that is locally generated in a power grid may cause severe faults in the connected high-security facilities, such as nuclear plants; critical failures in expensive, high-precision equipment (or devices); power outages; or even blackouts. In a smart grid (SG), where diverse renewable energy sources, such as solar, wind, and ocean waves, are interconnected and traded, reliable and accurate PQD feature detection is critical for sustainable source provision and grid failure prevention.

Each PQD event in an SG might originate from different sources. For instance, voltage sag can be induced by factors such as a short circuit, start of motor, faults in circuits, and switching of capacitors. Voltage swell may occur due to factors such as a single-line-toground fault and large load de-energization. Various nonlinear circuits or loads connected to the grid may induce harmonic currents. Fault-clearing or utility-switching often creates events such as interruption and transient signals. 
A wavelet transform modulus (WTM) with wavelet decomposition (WD) is often used to detect or localize the discontinuities of transient signals, such as images and PQDs [1,2]. Liao et al. [1] introduced a PQD detection scheme using the spatial correlation between wavelet coefficients (WCs), which further strengthens the edge points of a PQD signal compared to other signal points. However, this direct spatial correlation (i.e., direct intermultiplication between WCs) scheme has a limited (two or three) degrees of freedom due to the nature of WD; hereinafter, a dyadic discrete WD or wavelet packet decomposition (WPD) is assumed.

For better detection of the edge points of a PQD event signal (i.e., starting and ending edge points), $\mathrm{PQD}$ feature strengthening, together with wavelet (or wavelet packet)-based denoising, is often used. Several denoising techniques developed so far use S-transform [3], WT [4], mathematical morphology (MM) [5], and empirical mode decomposition (EMD) [6]. Recently, Bhuiyan et al. [7] proposed a localization system using the majority voting (MV)based interscale and intrascale indirect correlation metrics of WPCs to detect PQDs. They argued that the inherent additive white Gaussian noise (AWGN) in the power transmission system corrupts the detection capability; thus, denoising prior to PQD localization is required to further strengthen the PQD edge points. However, the simulation results indicate that the conventional wavelet or wavelet packet-based PQD feature detection is also highly sensitive to random (start or end) edge occurrence (i.e., random phase offset $\varphi_{m}$, which is uniformly distributed $\sim U[0,2 \pi)$ ) owing to the shift variance characteristics [8].

This paper proposes a wavelet packet transform modulus (WPTM) using WPD and a WPTM-based PQD detection scheme that is applicable to reliable smart grid construction. In addition, as the detection metric, we define (and use) the MV-based indirect correlation or root mean square (RMS). While WD decomposes only a low-frequency component (approximation) into two subcomponents (i.e., two WCs), WPD decomposes both low-frequency (approximation) and high-frequency (detail) components into two subcomponents (i.e., two wavelet packet coefficients (WPCs)). Hence, in contrast to the existing WTM-based direct correlation metrics [1], the proposed WPTM-based indirect correlation metrics exhibit an exponentially increased degrees of freedom (i.e., $D o F=P=2^{l}-1$ at level $l$ (except approximation)).

The impulsive noise that is mainly generated by the power switch ON/OFF or manmade electromagnetic interference (EMI) substantially hinders the PQD detection or localization [2]. However, it is not easily removable by using wavelet denoising alone, because of its broadband characteristics; thus, the PQD detection performance is considerably degraded. Throughout this paper, this impulsive noise disturbing wavelet- or wavelet packet-based PQD signal detection is differentiated from the transient events defined by the IEEE standard [9]. In particular, the presented PQD detection algorithm has an adaptive impulsive denoising step that makes it more robust to real channel conditions, including impulsive noise, than conventional schemes. Specifically, in the adaptive impulsive denoising step, if the signal sample is above a threshold $T H$ (= three-sigma level), a standard median (SM) plus WT-based denoising filter that can efficiently remove both impulsive noise and AWGN is chosen. Otherwise, a WT-based denoising filter that can only remove AWGN is chosen [10]. Numerical results prove that the presented WPTM-based PQD detection algorithm with larger degrees of freedom not only improves the correlation characteristics but is also more robust to random phase $\varphi_{m}$, impulsive noise, and low signal-to-noise ratio (SNR $\leq 25 \mathrm{~dB}$ ). Moreover, it also shows that the proposed algorithm with a threshold modification step reduces the false edge detection rate (FER) under such critical channel conditions.

The main contributions of this paper are listed as follows:

1. We define WPTM using WPC and propose a PQD feature detection (or localization) algorithm using WPTM-based metrics (such as RMS and MV). Hence, the presented WPTM-based PQD detection scheme, with larger degrees of freedom $\left(D o F=2^{l}-1\right)$, exhibits better correlation properties than the existing WTM-based PQD detection schemes (DoF $=$ two or three). 
2. For the WPTM-based PQD detection, we also define and use a modified threshold $\left(\mathrm{TH}_{\mathrm{M}}\right)$ rather than a conventional universal threshold $\left(\mathrm{TH}_{U}\right)$, which makes it more robust to practically severe power signal conditions, including random phase offset and low $\mathrm{SNR}(\leq 25 \mathrm{~dB})$

3. In the presented PQD detection procedure [see the details in Section 2.2], we add the SM plus wavelet filter-based adaptive denoising step after the pure power signal removal process (which follows the PQD feature strengthening process [7]), the socalled preprocessing stage, that effectively removes both impulsive noise and AWGN. Next, the proposed PQD detection stage using MV or RMS metric is executed. In particular, the MV metric uses the MV-based indirect correlation between WPCs instead of the conventional direct correlation between WCs.

4. A numerical analysis is performed for the proposed scheme (Section 3) under random power signal conditions and compared with the existing schemes. For reliable PQD localization, double edge-based detection is used and compared with the conventional single edge-based detection. In the numerical evaluation, various standard PQD event signals, including voltage sag, voltage swell, interruption, harmonics, notch, and so on, are generated and tested based on IEEE-1159. In addition, we implement a power distribution network using MATLAB ${ }^{\circledR}$ Simulink ${ }^{\circledR}$ (Natick, MA, USA) to create several PQD events and test the proposed scheme for the PQD signals generated by the network, for further validating our proposed system model. Via the numerical analysis, we prove that the proposed scheme is superior to the conventional waveletbased, wavelet-correlation-based, or non-wavelet-based schemes.

\subsection{Related Work}

In the literature, diverse PQD feature detection schemes using Fourier transform (FT), Short-time Fourier transform (STFT), phased-locked loop (PLL), Hilbert-Huang transform (HHT), S-transform, WT [1,4,11,12], and WPT [7,13] have been proposed.

FT extracts the frequency components of $\mathrm{PQD}$ event signals but does focus on the analysis of stationary signals [10]. STFT, also termed as windowed FT, overcame the limitations of FT by segmenting the signal into narrow time intervals; however, it cannot simultaneously provide suitable frequency and time resolutions [14]. In [15], PLL-based PQD detection was proposed; however, a small distortion of the mains voltage substantially degraded the detection performance. The authors in [3] presented an S-transform-based technique, where the denoising threshold was determined with the energy congregation level; however, this technique incurred a highly complex noise level estimation. In [16], the authors introduced HHT to detect and analyze PQDs, where MM was used to suppress the noise level. However, HHT is sensitive to the noise superimposed on an electric signal [17].

WT allows simultaneous signal localization in the time and frequency domains, which is a favorable characteristic for the analysis and detection of nonstationary transient signals $[1,4,18,19]$, such as PQD, as compared to the other schemes mentioned above, including FT and STFT $[10,14]$. Recently, Liao et al. showed that the spatial correlation between adjacent WCs is robust to nonlinear PQD signals over power signal channels [1]. WPT, which is an extended version of WT, allows the further decomposition of both high-frequency components (details) and low-frequency components (approximations) [7,13]. As the WPT output (i.e., WPCs) has larger degrees of freedom than the WT output (i.e., WCs), feature detection using WPCs exhibits a better spatial correlation property than waveletcorrelation-based-detection (WC-Det). Very recently, in [7], the authors presented a feature detection scheme using an MV-based indirect correlation. However, they used a universal threshold $T H_{U}$ for feature detection, which yields a large FER over severe power signal channels with random phase offset and impulsive noise.

In the present study, we extend our previous study [20] further by proposing an updated PQD detection algorithm that includes a preprocessing stage to make it more robust to severe power channel conditions like random phase $\varphi_{m}$ and low signal-to-noise ratio. Furthermore, we elaborate our previous study by adding an adaptive impulsive 
denoising function to the proposed algorithm, and thoroughly analyzing the presented system model under impulsive electricity channel conditions. With the help of numerical analysis, the proposed system model is proved to be superior to the conventional waveletbased, wavelet-correlation-based, or non-wavelet-based schemes.

The remainder of this paper is organized as follows. In Section 2, we briefly introduce the PQD signal model, address the proposed detection system model with adaptive denoising, explain WPT including how to decompose a PQD signal into different WPCs, define WPTM and WPTM-based metrics (MV and RMS), and present WPTM-based PQD feature detection using MV or RMS metric. Section 3 presents the numerical results of the proposed system model and a discussion on these results. Section 4 concludes the paper.

\section{PQD Feature Detection System Model Using WPTM-Based Metrics}

\subsection{Signal Model}

The occurrence of a single PQD event in a power grid can be a major cause of internal (or external) grid failure or severely damage various precision instruments, devices, equipment, and factories using the grid energy. Table 1 lists the several major PQD events that may often occur within the grid, such as sag, swell, harmonic, interruption, and notch.

Table 1. Power quality disturbance (PQD) event signal models and parameters.

\begin{tabular}{|c|c|c|}
\hline $\begin{array}{l}\text { PQD } \\
\text { Events }\end{array}$ & PQD Event Signal Models & Parameters \\
\hline Sag & $\begin{array}{c}s(t)=s_{0}(t)+q(t) \\
q(t)=-\alpha A\left(u\left(t-\tau_{1}\right)-u\left(t-\tau_{2}\right)\right) \sin (\omega t) \\
\tau_{1}<\tau_{2}, \quad u(t)=1, \quad t \geq 0\end{array}$ & $\begin{array}{c}0.1<\alpha \leq 0.9 \\
0.5 T \leq \tau_{2}-\tau_{1} \leq 30 T\end{array}$ \\
\hline Swell & $\begin{array}{c}s(t)=s_{0}(t)+q(t) \\
q(t)=\alpha A\left(u\left(t-\tau_{1}\right)-u\left(t-\tau_{2}\right)\right) \sin (\omega t), \tau_{1}<\tau_{2} \\
u(t)=1, \quad t \geq 0\end{array}$ & $\begin{array}{c}0.1<\alpha \leq 0.8 \\
0.5 T \leq \tau_{2}-\tau_{1}<30 T\end{array}$ \\
\hline Harmonics & $\begin{array}{c}s(t)=s_{0}(t)+q(t) \\
q(t)=A\left(u\left(t-\tau_{1}\right)-u\left(t-\tau_{2}\right)\right) \sin (\omega t)\left[\alpha_{3} \sin (3 \omega t)+\alpha_{5} \sin (5 \omega t)\right]\end{array}$ & $\begin{array}{c}0 \leq \alpha_{3} \leq 0.2 \\
0 \leq \alpha_{5} \leq 0.02 \text { Steady state }\end{array}$ \\
\hline Interruption & $\begin{array}{c}s(t)=s_{0}(t)+q(t) \\
q(t)=-a A\left(u\left(t-\tau_{1}\right)-u\left(t-\tau_{2}\right)\right) \sin (\omega t)\end{array}$ & $\begin{array}{c}\alpha>0.1 \\
0.5 T \leq \tau_{2}-\tau_{1} \leq 3 \mathrm{sec}\end{array}$ \\
\hline Notch & $\begin{array}{c}s(t)=s_{0}(t)+q(t) \\
q(t)=-\alpha \operatorname{sign}(\sin (\omega t)) A\left(u\left(t-\tau_{1}\right)-u\left(t-\tau_{2}\right)\right)\end{array}$ & $\begin{array}{c}0.1<\alpha \leq 0.4 \\
0.01 T \leq \tau_{2}-\tau_{1} \leq 0.05 T \\
\text { Steady state }\end{array}$ \\
\hline $\begin{array}{l}\text { Swell or } \\
\text { Sag with } \\
\text { harmonics }\end{array}$ & $\begin{array}{c}s(t)=s_{0}(t)+q(t) \\
q(t)= \pm \alpha A\left(u\left(t-\tau_{1}\right)-u\left(t-\tau_{2}\right)\right) \sin (\omega t)\left[\alpha_{3} \sin (3 \omega t)+\alpha_{5} \sin (5 \omega t)\right], \tau_{1}<\tau_{2}\end{array}$ & $\begin{array}{c}0.1<\alpha \leq 0.9 \\
0.5 T \leq \tau_{2}-\tau_{1} \leq 30 T 0 \leq \alpha_{3} \leq 0.20 \leq \alpha_{5} \leq \\
0.02\end{array}$ \\
\hline $\begin{array}{l}\text { Oscillatory } \\
\text { transient }\end{array}$ & $\begin{array}{c}(t)=s_{0}(t)+q(t) \\
q(t)=A\left(\alpha * e^{-\frac{\left(t-\tau_{1}\right)}{\varepsilon}} * u\left(t-\tau_{1}\right)-u\left(t-\tau_{2}\right)\right) \sin (5 \omega t)+\sin (\omega t), \tau_{1}<\tau_{2}\end{array}$ & $\begin{array}{c}0.1<\alpha \leq 0.8 \\
0.5 T \leq \tau_{2}-\tau_{1} \leq 3 T \\
8 \mathrm{~ms} \leq \varepsilon \leq 40 \mathrm{~ms}\end{array}$ \\
\hline
\end{tabular}

A power signal delivered through a power cable can be represented as

$$
x(t)=s(t)+n(t)=s_{0}(t)+q(t)+n(t)
$$

where $s(t)\left(=s_{0}(t)\right.$ [pure power source] $+q(t)$ [distortion component]) is a PQD event signal and $n(t)$ is an additive noise signal.

In the absence of any PQD event, that is, $q(t)=0, s(t)$ represents the standard (pure) sinusoidal electric power signal, that is, $s(t)=s_{0}(t)=A \sin (\omega t)$, where the peak value of the power signal $A=220 \sqrt{2} \mathrm{~V}$ and its radian frequency $\omega=2 \pi f \mathrm{rad} / \mathrm{s}(f=1 / T$ [=electric signal period] $=60$ or $50 \mathrm{~Hz}$ ). However, when a PQD event occurs, that is, $q(t) \neq 0$, $s(t)=s_{0}(t)+q(t)$, which becomes one of the PQD event signal models listed in Table 1 [9]. In these signal models, $\tau_{1}$ and $\tau_{2}$ indicate the starting and ending edge points of the PQD events, respectively. The signal model parameters, such as $\tau_{1}, \tau_{2}$, and $\alpha$ (amplitude ratio), as well as the power cable noise component $n(t)$, can be intrinsically random. 
The additive noise $n(t)$ in (1) is the sum of the Gaussian noise component $n_{a}(t)$ and impulsive noise component $n_{i}(t)$ [2]:

$$
n(t)=n_{a}(t)+n_{i}(t)
$$

where the impulsive noise component $n_{i}(t)$, which mainly results from the power switching ON/OFF or man-made EMI, may severely hinder the PQD signal detection (or localization) [2]. In case of Gaussian noise, the typical magnitude of noise is less than 1\% (i.e., $\mathrm{SNR}=40 \mathrm{~dB}$ ) of the voltage magnitude [9].

For impulsive noise sample generation, we apply the following statistical Bernoulli Gaussian model [21]:

$$
n_{i}(t)=b(t) g(t)
$$

where $b(t)$ is the Bernoulli process with the probability of success $\psi$, and $g(t)$ is the Gaussian process with zero mean and variance $\sigma_{I}^{2}$.

After the A/D conversion, the noisy power signal $x(t)$ is converted into the following sampled version (assuming sampling interval $T_{S}$ ):

$$
x[i]=s[i]+n[i]=s[i]+n_{a}[i]+n_{i}[i],
$$

where $s[i]$ indicates the PQD signal samples, $n_{a}[i]$ indicates the Gaussian noise samples with zero mean and variance $\sigma_{G}^{2}$, and $n_{i}[i]$ indicates the impulsive noise samples with zero mean and variance $\sigma_{I}^{2}$. The two variances $\sigma_{G}^{2}$ and $\sigma_{I}^{2}$ are related by the impulsive to Gaussian power ratio $\mu=\sigma_{I}^{2} / \sigma_{G}^{2}$.

\subsection{System Model}

This paper proposes a PQD detection algorithm with adaptive impulsive denoising, whose flowchart is shown in Figure 1. In the proposed algorithm, if the noise type is "impulsive" $\left(N_{c}=1\right)$, we use both an SM filter [22], for removing the impulsive noise components $n_{i}[i]$, and a wavelet denoising filter, for removing the Gaussian noise components $n_{a}[i]$; otherwise (i.e., if the noise type is "Gaussian" $\left(N_{c}=0\right)$ ), we only use a wavelet denoising filter. In the classification of the noise type (see Step 3 in Figure 1), the noise type of the input signal is determined using a threshold $(t h)$. In this step, the 3-sigma threshold, that is, $t h=3 \sigma$, is used, where $\sigma$ is the standard deviation of the output samples (see $\hat{y}[i]$ in (6)) from Step 2. That is, $N_{c}=1$ if $\hat{y}[i] \geq t h$ and 0 if $\hat{y}[i]<t h$.

In what follows, the proposed algorithm procedure based on the flowchart shown in Figure 1 is explained in detail, where an $80 \%$ pu sag signal (Figure 2a), which is added with Gaussian plus impulsive noise (i.e., under impulsive noise channel (IMPN) conditions; the signal is shown in Figure 2b), is used as an example.

Step 1: Obtain the estimated (extracted) noise plus distortion component signal, denoted as $y[i]=q[i]+n[i]$ (Figure $2 \mathrm{c}$ ), by directly subtracting the power source signal $s_{0}[i]$ from the input signal $x[i]$, as follows:

$$
y[i]=x[i]-s_{0}[i]
$$

Step 2: Apply a wavelet-based denoising filter to $y[i]$ for the removal of Gaussian noise. As a result, we obtain a denoised (estimated) distortion component signal (Figure 2d), as follows:

$$
\hat{y}[i]=y[i]-\hat{n}_{a}[i] .
$$

Step 3: Classify the estimated distortion component signal into two noise types: impulsive noise type $\left(N_{c}=1\right)$, if the output samples of Step 2 are above 3-sigma, and otherwise, non-impulsive type $\left(N_{c}=0\right)$. If $N_{c}=1$, then go to Step 4; otherwise, go to Step 5. 
Step 4: Use the SM filter for impulsive noise removal, whose output becomes (Figure 2e)

$$
\widetilde{y}[i]=\hat{y}[i]-\hat{n}_{i}[i]
$$

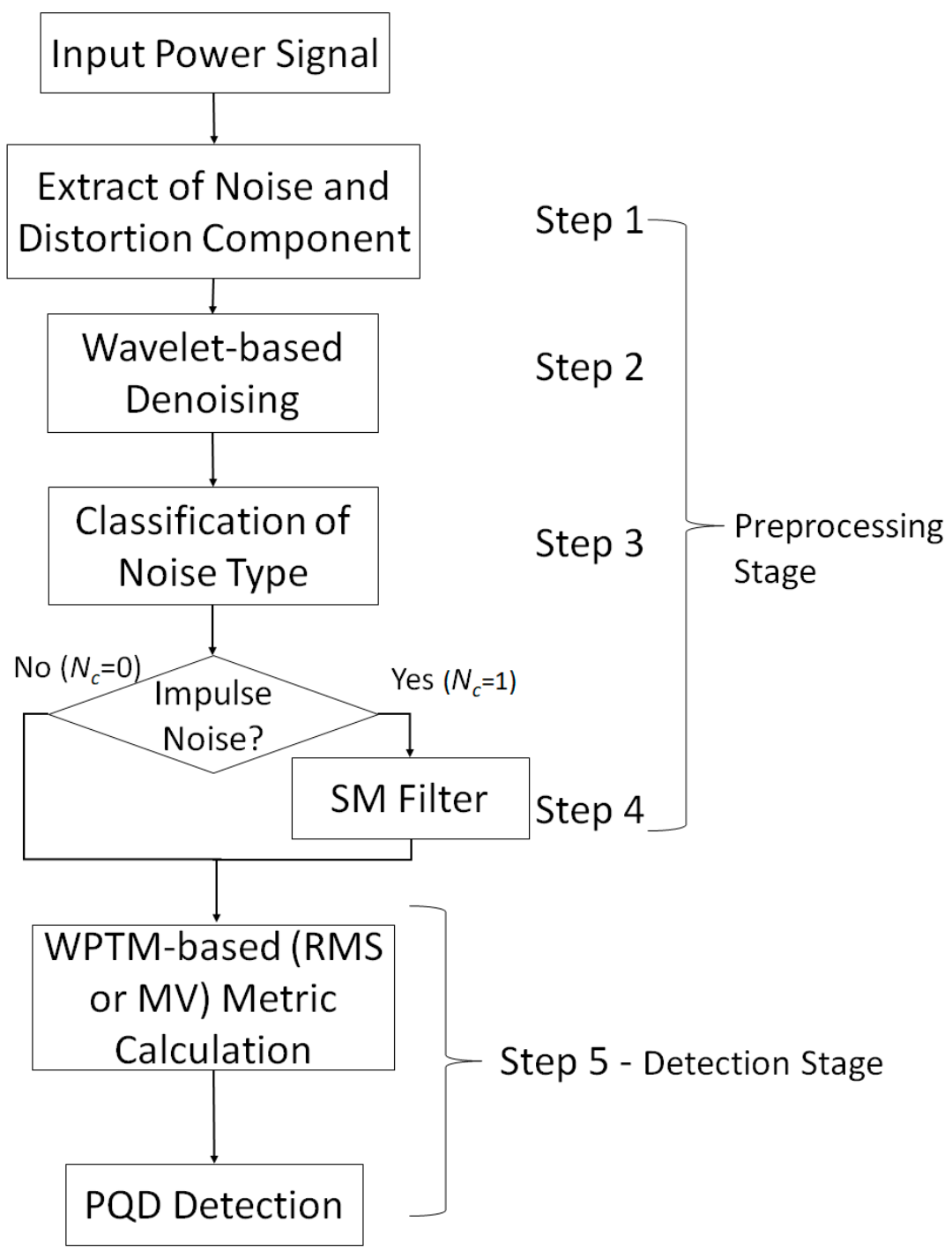

Figure 1. Flowchart of the proposed PQD feature detection with adaptive impulsive denoising.

Although this SM filter, which is a type of nonlinear filter, is simple, it has an excellent impulse noise cancelation function [22].

Step 5: Finally, execute the presented PQD localization process using WPTM metrics (whose pseudocode is presented in Algorithm 1 [for MV metric] or Algorithm 2 [for RMS metric]), which improves the robustness of the proposed PQD detection system over nonstationary noisy power signal channels [7].

In the wavelet-based Gaussian denoising step (Step 2), we choose a Daubechies wavelet with a five-coefficient filter (DB5), which is relatively better than the other Daubechies wavelet filters (e.g., three-coefficient (DB3), six-coefficient (DB6), or eight-coefficient (DB8)) in terms of the recovered SNR (for simplicity, the comparison of numerical results among these filters is omitted here). That is, in this Gaussian denoising step (Step 2), we first use DB5 with a universal threshold $t h=\sigma_{w} \sqrt{2 \log |N|}$ (where $N$ is the size of the WT sample vector $\mathbf{W}_{l}^{1}$ and $\sigma_{w}=\operatorname{med}\left(\mathbf{W}_{l}^{1}\right)$ /0.6745) [9]. Then, if the estimated noise type in Step 3 is impulsive, that is, $N_{c}=1$, in Step 4, we use an SM filter that removes the impulsive noise. 


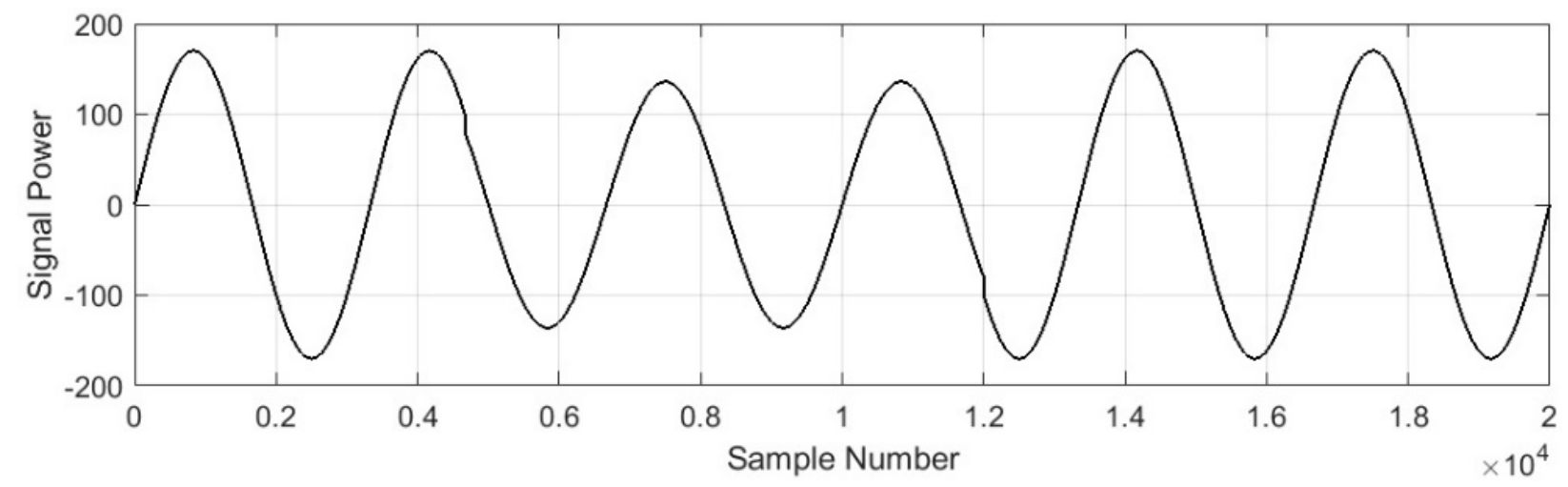

(a)

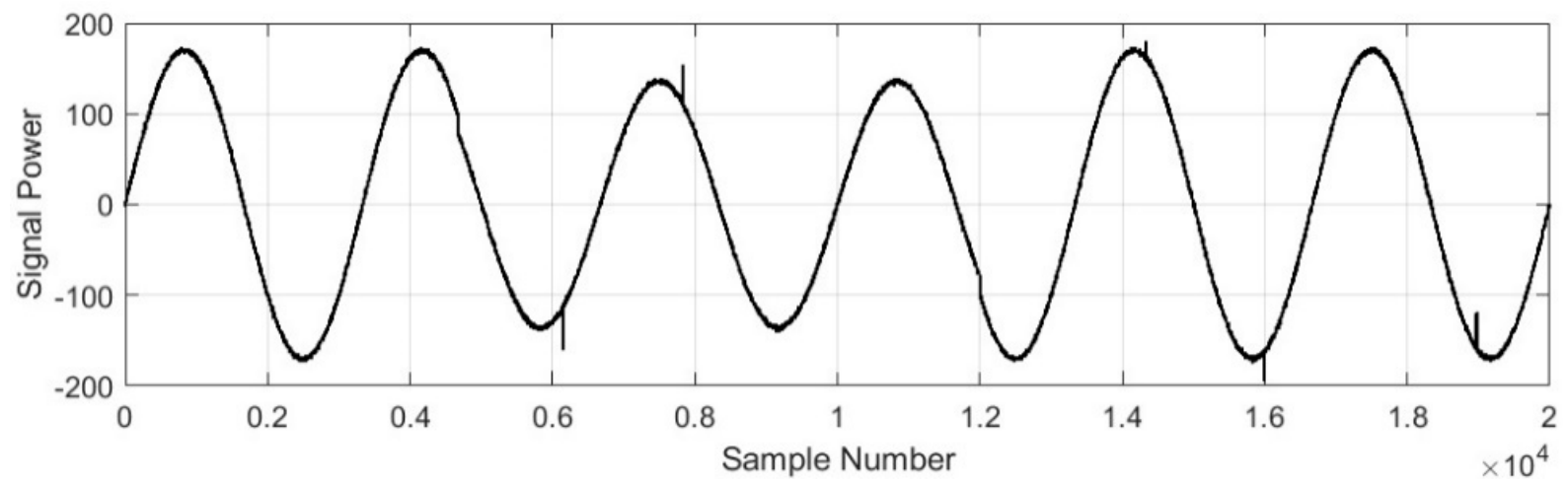

(b)

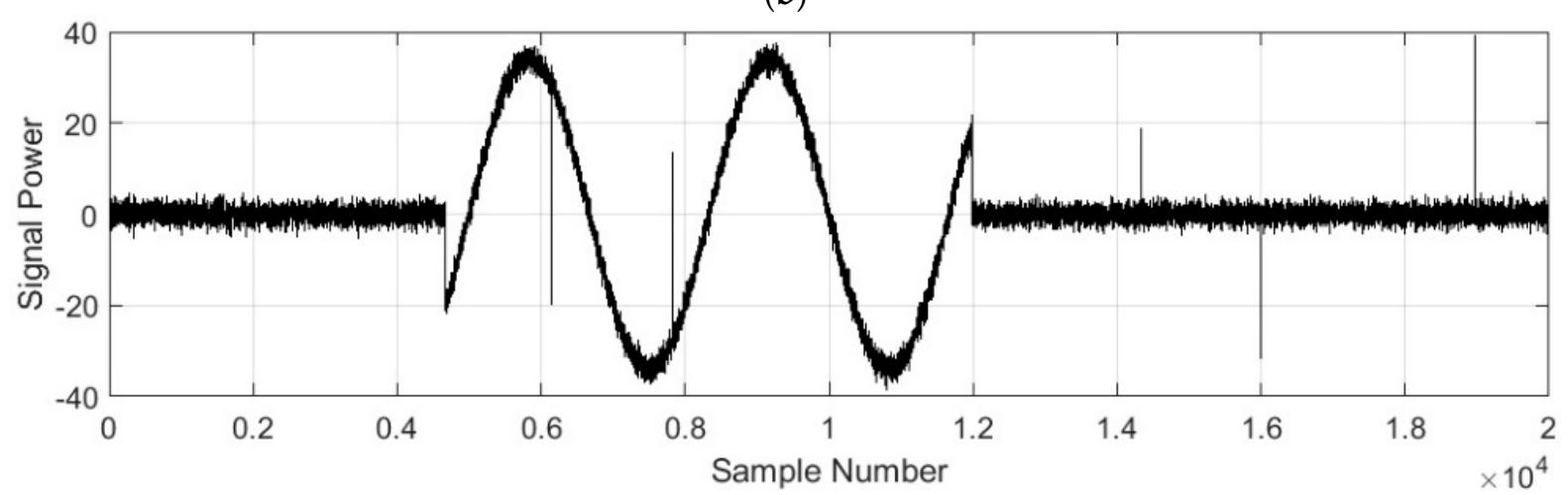

(c)

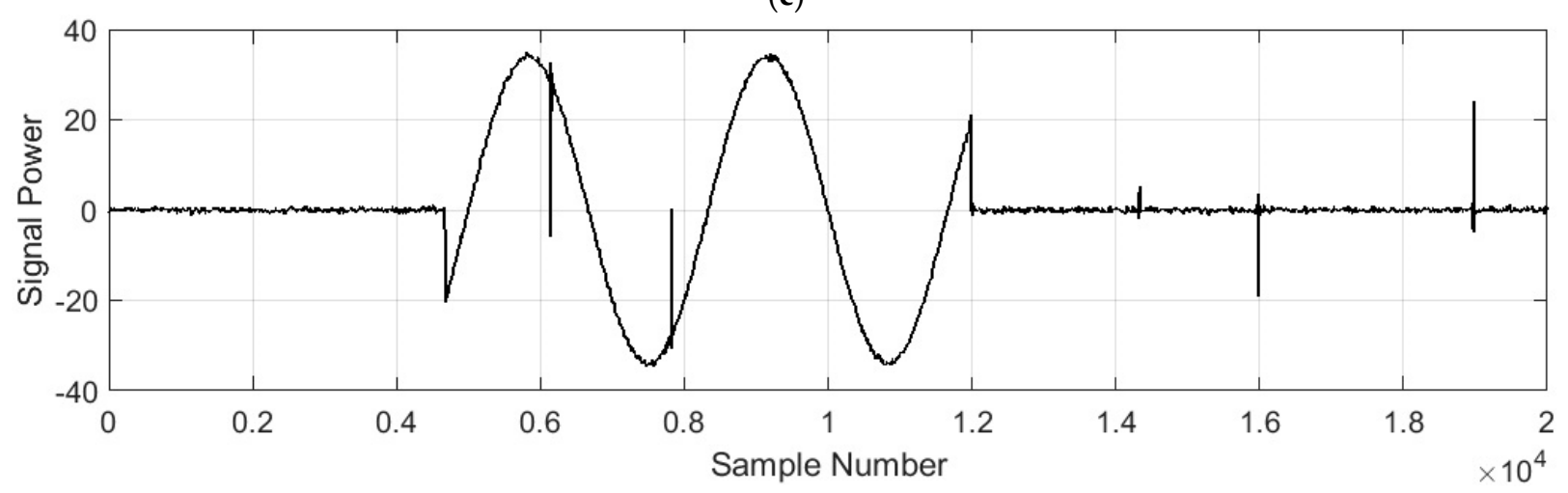

(d)

Figure 2. Cont. 


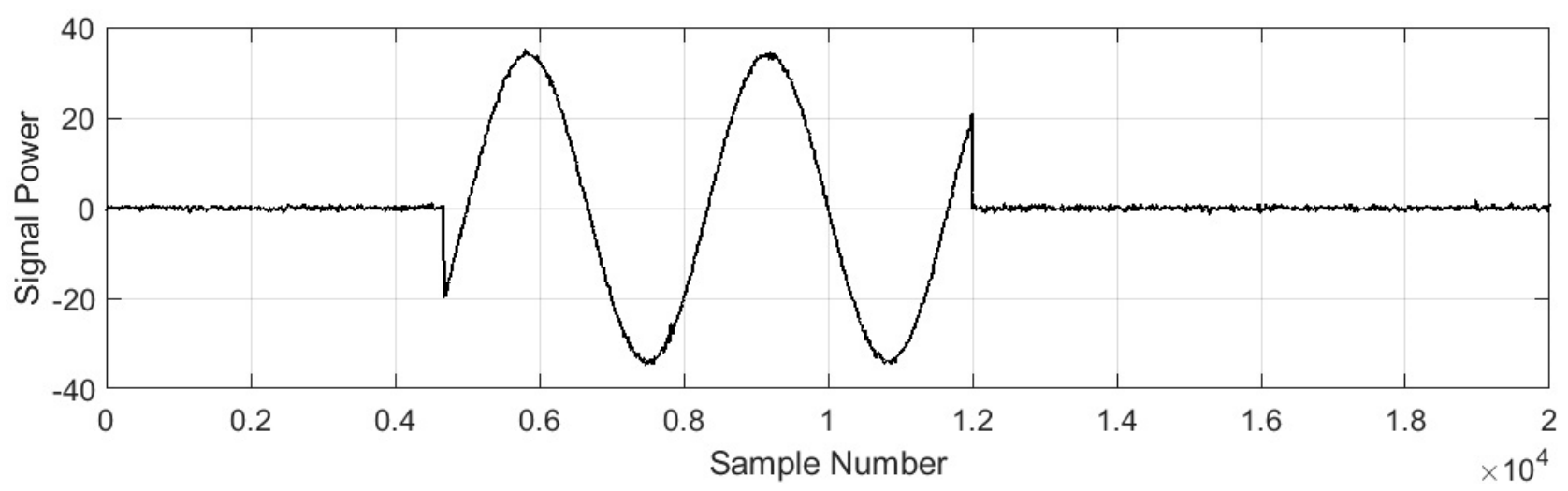

(e)

Figure 2. (a) Pure sag signal with $80 \% p u$, (b) sag signal with $80 \% p u$ over the Gaussian plus impulsive noise channel (where $\mu=400$, $\psi=0.02 \%$, and SNR $=35 \mathrm{~dB})$, (c) extracted noise plus distortion component signal $y[i],(\mathbf{d})$ wavelet-based Gaussian-denoised distortion component signal $\hat{y}[i]$, and (e) SM-filter-based impulsive-denoised distortion component signal $\widetilde{y}[i]$.

After the Gaussian and impulsive denoising process (Steps 2-4 in Figure 1), the WPTMbased PQD detection process (Step 5 in Figure 1) is performed, for which Daubechies's WPT with DB3 is assumed to be used. The presented PQD detection scheme using WPTM-based metric (MV or RMS) is detailed in the next subsection.

\subsection{PQD Feature Detection Using WPTM-Based Metrics \\ 2.3.1. WPT}

WPT generates WPCs through WPD, whereas WT generates WCs through WD. Throughout the paper, a dyadic WD or WPD is assumed to be used (Figure 3), where the input signal vector, denoted as $\mathbf{W}_{0}^{0}=\mathbf{V}_{0}=\{x[i] \mid i=0,1, \ldots, I-1\}$, is the root node of the binary tree at the level index $l=0$. Using a level- $l(\geq 1) \mathrm{WD}$, the approximation vector $\mathbf{W}_{l-1}^{0}$ at the previous level (i.e., level $l-1$ ) is recursively projected into two level- $l$ orthonormal wavelet basis functions, that is, a scale function $\phi_{l}^{0}\left(=\psi_{l}^{0}\right)$ and a wavelet function $\psi_{l}^{1}$, which generate the correspondingly decomposed two wavelet vectors (also termed as WCs): approximation (low frequency) $\mathbf{W}_{l}^{0}$ and detail (high frequency) $\mathbf{W}_{l}^{1}$. In contrast, using the level-l WPD, both approximation (low frequency) $\mathbf{W}_{l-1}^{0}$ and details (high frequency) $\mathbf{W}_{l-1}^{p}$ (where $p=1, \ldots, 2^{l-1}-1$ ) at the previous level (i.e., level $l-1$ ) are recursively projected into the level-l orthonormal WP basis functions $\psi_{l}^{p}$ (where $p=0,1, \ldots, 2^{l}-1$ ) [23], thus producing the correspondingly decomposed $2^{l}$ wavelet packet vectors $\mathbf{W}_{l}^{p}$ (which are also called WPCs). Hence, as observed in the binary tree of Figure 3, while WD decomposes the original input signal vector $\mathbf{W}_{0}^{0}$ into $l+1$ WCs (e.g., for $l=3, \mathbf{W}_{0}^{0}=\left[\mathbf{W}_{3}^{0}\left|\mathbf{W}_{3}^{1}\right| \mathbf{W}_{2}^{1} \mid \mathbf{W}_{1}^{1}\right]$ ), WPD decomposes $\mathbf{W}_{0}^{0}$ into $2^{l}$ WPCs (e.g., for $l=3, \mathbf{W}_{0}^{0}=\left[\mathbf{W}_{3}^{0}\left|\mathbf{W}_{3}^{1}\right| \ldots \mid \mathbf{W}_{3}^{7}\right]$ ).

In WPT, we select the "cost function" in each WP sub-band as an optimal representation basis of the input signal. The cost function can determine the cost value of each node and its children nodes in the obtained full binary tree (Figure 3). There are several possible choices for the cost function, such as 1-norm, Shannon entropy (SE), and log energy. Of these, we choose the Shannon entropy of the coefficients of sub-band $S$, which is calculated as follows [2]:

$$
S E(S)=-\sum_{i} S_{i}^{2} \log \left(S_{i}^{2}\right) .
$$

Liao et al. showed that the spatial correlation between WCs can emphasize the starting and ending points of PQDs and suggested a wavelet-correlation-based detection method that improves the PQD detection performance over that of generic wavelet-based denoising methods [1]. However, this scheme has limited degrees of freedom (DoF $=2$ or 3$)$ and covers a limited frequency range as $l$ increases, as WD decomposes only the approximation (low frequency) vector $\mathbf{W}_{l}^{0}$. 


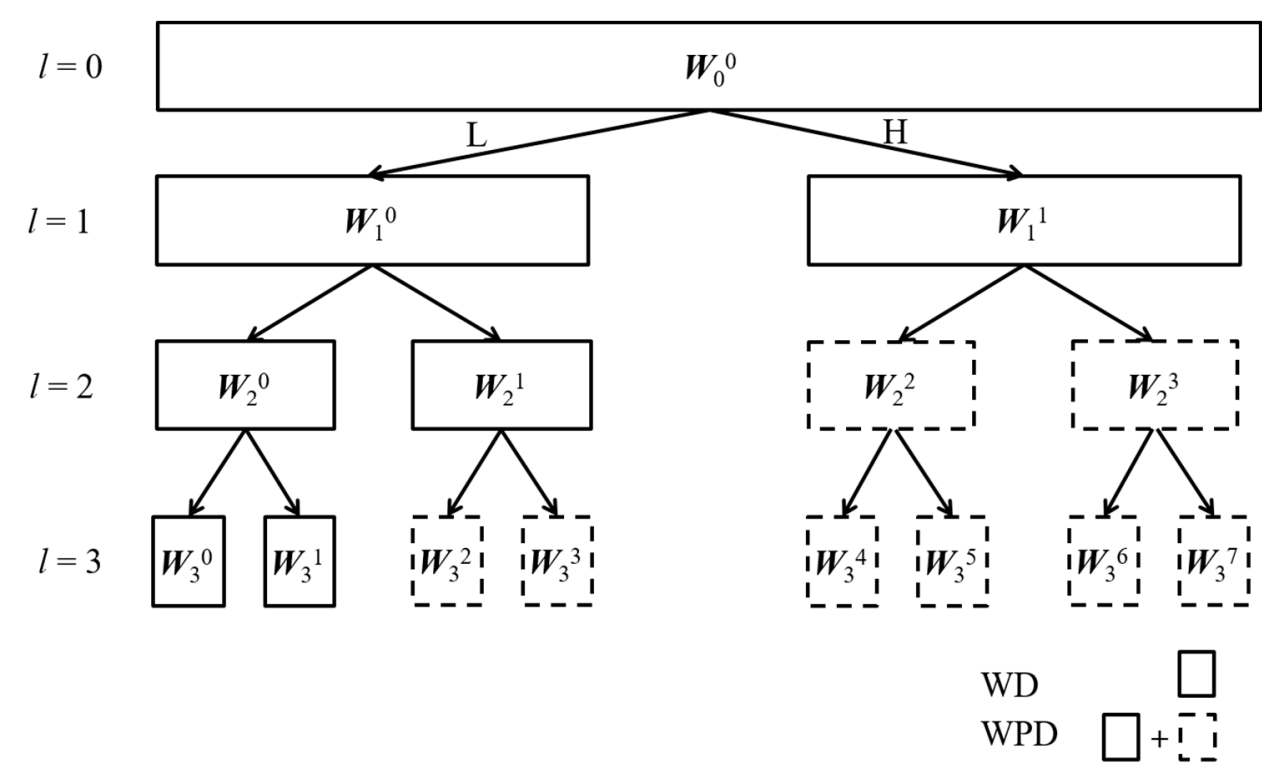

Figure 3. WD and WPD.

In contrast, this paper defines WPTM using WPCs, introduces the WPTM-based metrics that exhibit large degrees of freedom $\left(D o F=2^{l}-1\right)$, and present a PQD feature detection system that uses these WPTM-based metrics.

\subsubsection{WPTM and WPTM-Based Metrics}

WTM using WCs has been widely applied to locate (or detect) the discontinuities of transient signals, such as images [2]. This paper similarly defines WPTM using WPCs and suggests several WPTM-based metrics for reliable PQD feature detection under severe power signal conditions.

In a binary tree, as shown in Figure 3, the top node (i.e., top WPC) is $\mathbf{W}_{0}^{0}=V_{0}=$ $\{x[i] \mid i=0,1, \ldots, I-1\}$. A WP space $\mathbf{W}_{l}^{p}$ (where the level index $l=0, \ldots, L-1$ and the packet index $p=0, \ldots, 2^{l}-1$ ) is defined with its corresponding orthonormal basis functions $\psi_{l}^{p}$. By using the dyadic WPD, a WP space $\mathbf{W}_{l}^{p}$ is decomposed into two WP subspaces (producing the corresponding two WPCs) $-\mathbf{W}_{l}^{p}=\mathbf{W}_{l+1}^{2 p} \oplus \mathbf{W}_{l+1}^{2 p+1}$-which are, respectively, defined with their corresponding basis functions $\psi_{l}^{p}$ as follows:

$$
\begin{gathered}
\psi_{l+1}^{2 p}[i]=\sum_{n=-\infty}^{\infty} h[n] \psi_{l}^{p}[i-r], \\
\psi_{l+1}^{2 p+1}[i]=\sum_{n=-\infty}^{\infty} g[n] \psi_{l}^{p}[i-r], \\
h[n]=\left\langle\psi_{l+1}^{2 p}[u], \psi_{l}^{p}[u-r]\right\rangle, \\
g[n]=\left\langle\psi_{l+1}^{2 p+1}[u], \psi_{l}^{p}[u-r]\right\rangle
\end{gathered}
$$

where $r=2^{l} n$ denotes the translation index and $\psi_{l}^{p}$ can be represented with $2^{l}$ elementary basis functions $\psi^{p}[\cdot]: \psi_{l}^{p}[i-r]=\frac{1}{\sqrt{2^{l}}} \psi^{p}\left[\frac{i-r}{2^{l}}\right], p=0, \ldots, 2^{l}-1$.

Let us assume that we construct an admissible tree for the input signal $x[i]$ with all $l$ th-level wavelet basis functions $\psi_{l}^{p}$. Then, the corresponding WPCs of $x[i]$ become

$$
\boldsymbol{W}_{l}^{p}=\left\{W_{l}^{p}[n] \mid n=1, \ldots, N\right\},
$$


where $W_{l}^{p}[n]=\left\langle x[i], \psi_{l}^{p}[i-r]\right\rangle=\sum_{i=0}^{I-1} x[i], \psi_{l}^{p}[i-r]$ and $x[i]=\sum_{p=0}^{2^{l}-1} \sum_{i=0}^{I-1}\langle x[i]$, $\left.\psi_{l}^{p}[i-r]\right\rangle \psi_{l}^{p}[i-r]$.

Considering the transient (high-frequency) component of the PQD signal $x[i]$, we can approximate the component with all WPCs besides approximation $\left(\mathbf{W}_{l}^{0}\right)$. Hence, for level $l$, the WPC matrix $\mathbf{W}$ of the signal $x[i]$ can be approximately expressed with $P=2^{l}-1$ WPCs (except approximation) as follows:

$$
\mathbf{W}=\left[\begin{array}{c}
\mathbf{W}_{l}^{1} \\
\vdots \\
\mathbf{W}_{l}^{P}
\end{array}\right]=\left[\begin{array}{ccc}
W_{l}^{1}[1] & \cdots & W_{l}^{1}[N] \\
\vdots & \ddots & \vdots \\
W_{l}^{P}[1] & \cdots & W_{l}^{P}[N]
\end{array}\right]
$$

For level $l$, WPTM exhibits an exponentially increasing degrees of freedom, that is, $D o F=P=2^{l}-1$, whereas WTM exhibits a linearly increasing degrees of freedom, that is, $D o F=2$ or 3 (except approximation) [1]. Furthermore, while WTM has a limited spectrum support (only low-frequency part), due to the nature of WD, WPTM maintains the entire spectrum support, due to the nature of WPD (Figure 3). Hence, WPTM exhibits better correlation properties than WTM while appropriately retaining the high-frequency components of the PQD signal.

We can also specify the basis functions $\psi_{l}^{p}$ with $\theta_{l}(\boldsymbol{u})=2^{-l} \theta\left(2^{-l} \boldsymbol{u}\right)$ (where $\boldsymbol{u}=\left\{u_{p} \mid p=1, \ldots, P\right\}$ indicates the orthonormal vectors defining the wavelet packet signal space and $\theta(\cdot)$ is the convolution kernel), where conventional WTM-based schemes have been used for the edge detection of nonstationary signals, such as images [2]. In this study, for PQD detection, we use scaled wavelets, denoted by

$$
\bar{\psi}_{l}^{p}=2^{l} \frac{\partial \bar{\theta}_{l}}{\partial u_{p}},
$$

which can smoothen the PQD signal space surface. In (12), $\bar{\psi}_{l}^{p}(\boldsymbol{u})=\psi_{l}^{p}(-\boldsymbol{u})$ and $\bar{\theta}_{l}(\boldsymbol{u})=$ $\theta_{l}(-\boldsymbol{u})$. Then, the $l$ th-level WPT components (i.e., WPCs) for the PQD input signal $x[i]$ can be redefined with $\bar{\theta}_{l}$ as follows:

$$
W_{l}^{p}[n]=\left\langle x[i], \psi_{l}^{p}[i-r]\right\rangle=\frac{\partial}{\partial u_{p}}\left(x * \bar{\theta}_{l}\right)(u),
$$

where $n$ and $r=2^{l} n$ are the time-shift and translation parameters, respectively.

Thus, we rewrite the WPC matrix $\mathbf{W}$ in (11) with the gradient vector smoothened by $\bar{\theta}_{l}$, as follows:

$$
\mathbf{W}=\left[\begin{array}{c}
\mathbf{W}_{l}^{1} \\
\vdots \\
\mathbf{W}_{l}^{P}
\end{array}\right]=2^{l}\left[\begin{array}{c}
\frac{\partial}{\partial u_{1}}\left(x * \bar{\theta}_{l}\right)(\boldsymbol{u}) \\
\frac{\partial}{\partial u_{2}}\left(x * \bar{\theta}_{l}\right)(\boldsymbol{u}) \\
\vdots \\
\frac{\partial}{\partial u_{2^{l}-1}}\left(x * \bar{\theta}_{l}\right)(\boldsymbol{u})
\end{array}\right]=2^{l} \nabla\left(x * \bar{\theta}_{l}\right)(\boldsymbol{u}) .
$$

Hereinafter, for simplicity, we will drop the subscript $l$ of the WPC $\mathbf{W}_{l}^{p}$ in (11) and (14).

Throughout the paper, for PQD feature detection, we define the WPTM $M$ as a function of the $\mathbf{W}$ components, as follows:

$$
\mathbf{M}=\left\{M[n]=f\left(\left|W^{1}[n]\right|,\left|W^{2}[n]\right|, \ldots,\left|W^{P}[n]\right|\right) \mid n=1, \ldots, N\right\} .
$$

From (15), it is confirmed that the presented WPTM-based metrics exhibit greater degrees of freedom $\left(P=2^{l}-1\right)$ than the existing wavelet-based or wavelet-correlation-based metrics. Thus, the proposed scheme exhibits improved PQD signal detection and local- 
ization capability compared to the existing schemes [1]. This difference further increases under critical channel conditions, such as low SNR $(\leq 25 \mathrm{~dB})$.

Conventionally, considering only the Gaussian noise channel (AWGN), the PQD feature detection threshold th of each WPC can be initialized with the following universal threshold $T H_{U}[24]$ :

$$
T H_{U}=t h=\sigma_{d}^{p} \sqrt{2 \log |N|},
$$

where the parameter $\sigma_{d}^{p}$ is the median of $\mathbf{W}^{p}$; that is,

$$
\sigma_{d}^{p}=\frac{\operatorname{med}\left(\mathbf{W}^{p}\right)}{0.6745}
$$

The PQD event is inherently random and its channel characteristics are not pure Gaussian. In particular, the numerical results (Section 3) indicate that the PQD edge detection (or localization) is much sensitive to random phase offset $\varphi_{m}=\left\{\tau_{m} \bmod T\right\} \times$ $2 \pi / T$, where $\varphi_{m} \sim U[0,2 \pi)$ is uniformly distributed and $\mathrm{m}=1$ and 2 indicate the start and end edge locations, respectively, because of the shift variant characteristics of the wavelets or wavelet packets. When assuming the use of the universal threshold $T H_{U}$, Figure 4 shows the average detection rate (DR) and FER curves of a sag signal with $80 \%$ $p u$ (assuming Gaussian noise with SNR $=35 \mathrm{~dB}$ ) along the phase offset axis. DR can be almost $0 \%$ at approximately $\varphi_{m} \approx \pi / 2$ or $3 \pi / 2$. This means that even after the denoising step (see Steps 2-4 in Figure 1), $T H_{U}$ [7] does not work well, especially for the detection of a random $\mathrm{PQD}$ event signal. The numerical results also prove that FER is very high when using $\mathrm{TH}_{U}$ (Figure 4 and Table 2).

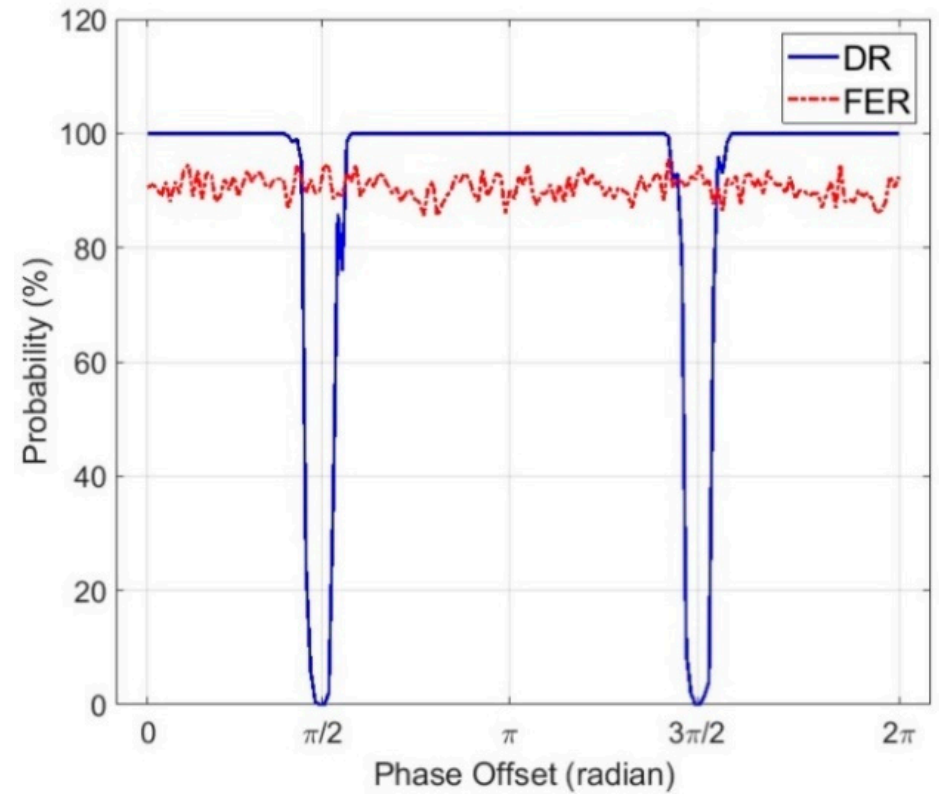

Figure 4. Detection rate (DR) and false edge detection rate (FER) versus phase offset $\varphi_{m}$ (assuming $\mathrm{SNR}=35 \mathrm{~dB}$ ), where a universal threshold-based detection is used.

Table 2. Performance comparison (DR/FER (\%)) of the proposed MV-based detection scheme for fixed and random PQDs, for which a sag signal of $80 \%$ pu is generated over Gaussian noise channel with $\mathrm{SNR}=40 \mathrm{~dB}$.

\begin{tabular}{ccc}
\hline & Fixed PQD & Random PQD \\
\hline$T H_{U}$ & $100 / 87.8$ & $91.8 / 90.5$ \\
$T H_{M}(k=1.4)$ & $100 / 0.0$ & $88.9 / 8.5$ \\
\hline
\end{tabular}


Hence, to avoid such high FER, we define a modified threshold with " $k$ " factor (Table 3), whose value depends on the used metric, such as MV or RMS. In the proposed algorithm, an optimal value of $k$ that maintains a low FER $(<12 \%)$ without damaging the DR (even if it remains low at $\varphi_{m} \approx \pi / 2$ or $3 \pi / 2$ ) is obtained through simulation. Consequently, the modified threshold $\left(\mathrm{TH}_{\mathrm{M}}\right)$ of the proposed scheme is determined as

$$
T H_{M}=k \times T H_{U}=k \sigma_{d}^{p} \sqrt{2 \log |N|} .
$$

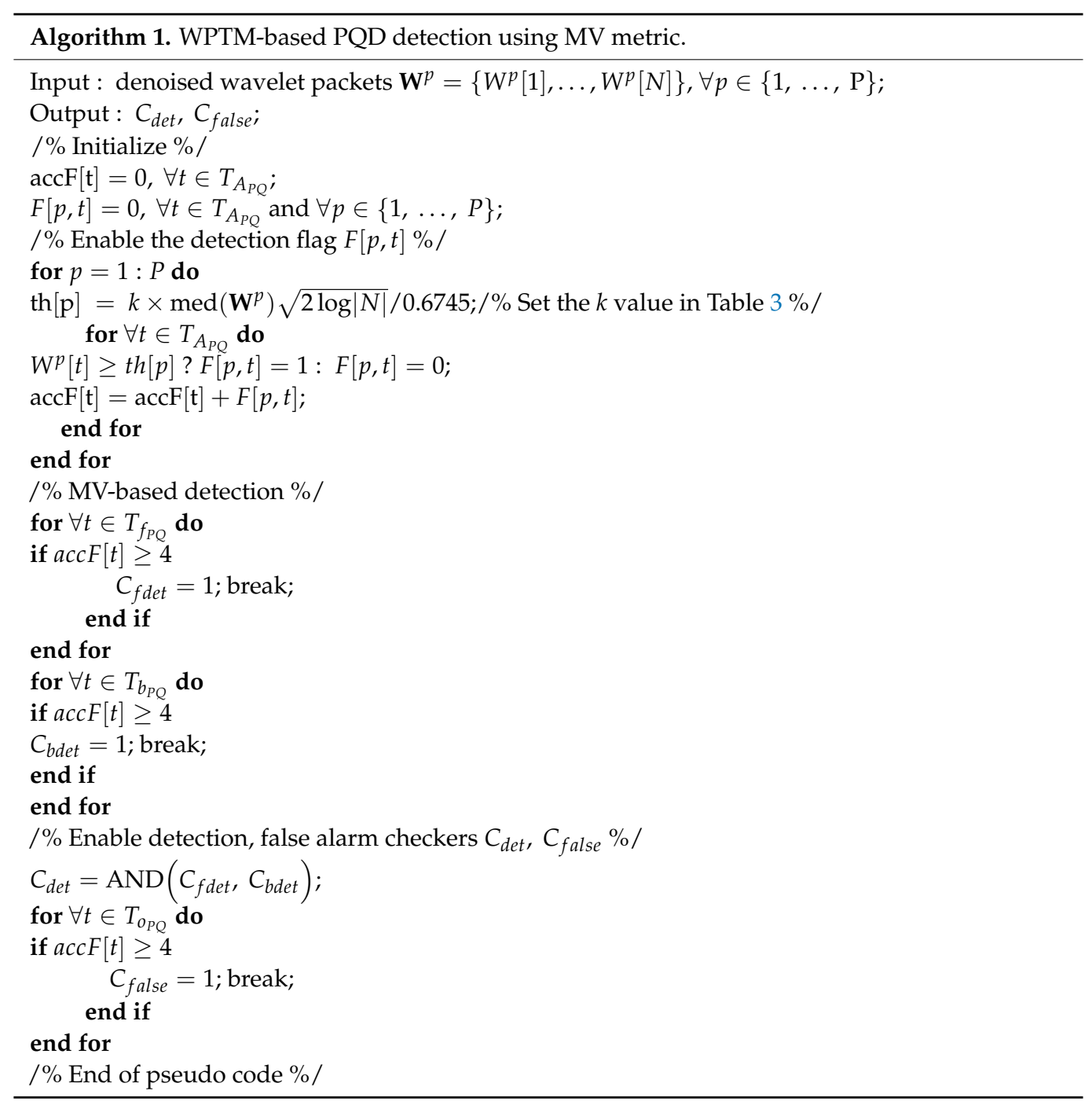

Table 3. Comparison of PQD metrics.

\begin{tabular}{|c|c|c|c|c|}
\hline & & MV & RMS & Conv. [1] \\
\hline \multicolumn{2}{|c|}{ Complexity } & Higher & Lower & Much Higher \\
\hline \multicolumn{2}{|c|}{ Strength } & Higher DR & Lower FER & $\begin{array}{c}\text { Lower DR \& } \\
\text { Higher FER }\end{array}$ \\
\hline \multirow{2}{*}{$k$ factor } & Gaussian Noise & 1.4 & 1.1 & - \\
\hline & Impulsive Noise & 1.6 & 1.4 & - \\
\hline
\end{tabular}




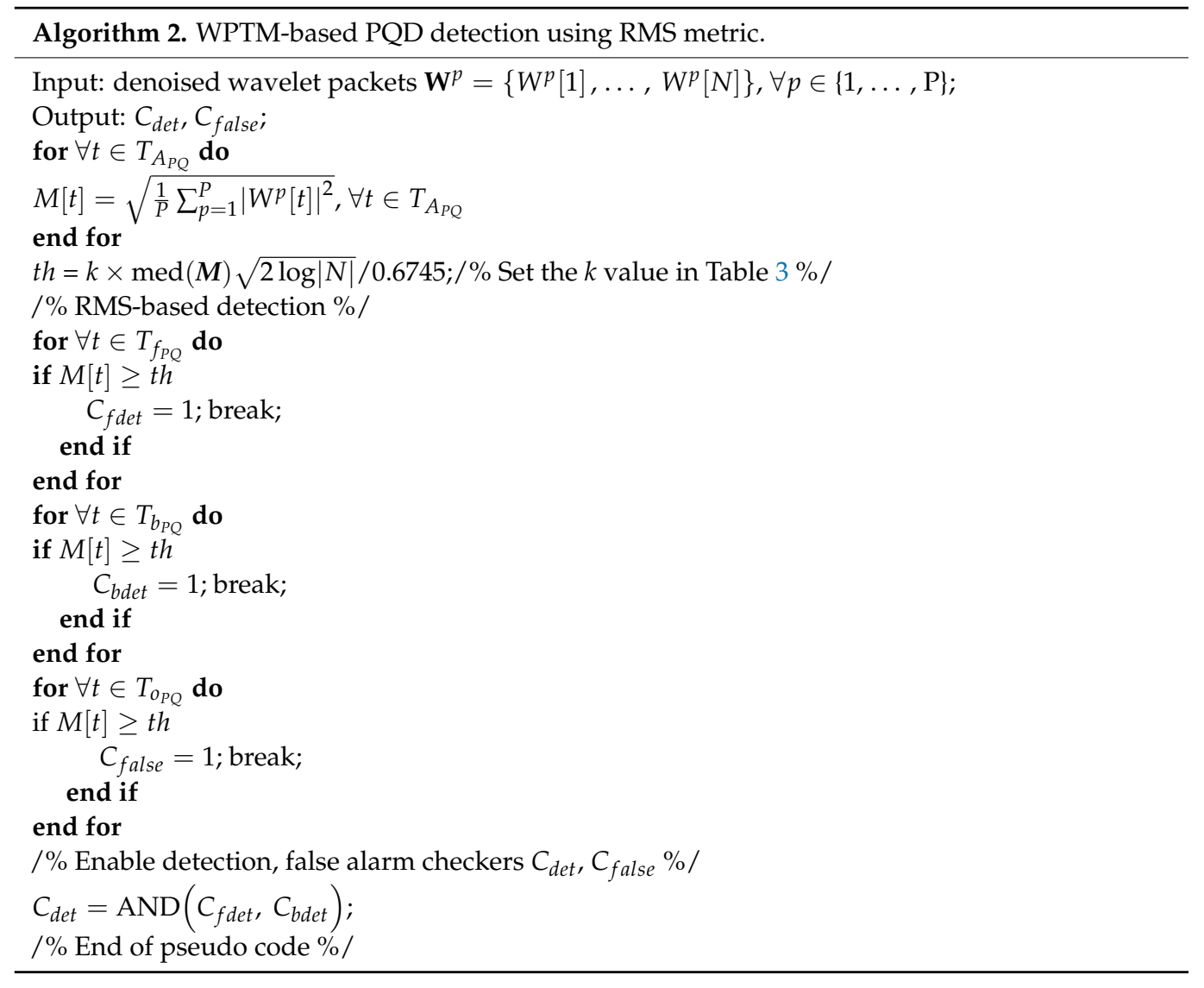

\subsubsection{PQD Feature Detection Using MV Metric}

We define an MV-based WPTM metric $\mathbf{M}=\mathbf{M}_{m v}$ and use it for PQD feature detection. This metric is based on MV-based indirect correlation rather than direct correlation in existing schemes [1]. While the existing metric using wavelet correlation exhibits a limited degrees of freedom ( $D o F=2$ or 3$)$, the proposed MV metric using indirect WP correlation exhibits an exponentially increased degrees of freedom (i.e., $D o F=P=2^{l}-1$ ). Hence, the MV metric is relatively simple yet robust to severe random PQD characteristics, including phase offset and impulsive noise.

For reliable PQD localization, we choose double-edge-based detection instead of the conventional single-edge-based detection [1,25], where we use two (front and back) edge checkers, denoted as $C_{f d e t}$ and $C_{\text {bdet }}$, such that a PQD event will be detected only if both edge checkers are enabled.

In the detection procedure presented in Figure 1, Step 5 can call the WPTM-based $\mathrm{PQD}$ detection function using the MV metric (Algorithm 1). In Algorithm 1, the refined (denoised) wavelet packets $W^{p}$ (where $p=1, \ldots, P$ ) that undergo the impulsive denoising process and wavelet-based Gaussian denoising process (see Steps 2 to 4 in Figure 1) are input. We assume that DB3 is used for WPD. In the MV-based detection procedure presented in Algorithm 1, we first enable (" 1 ") or disable (" 0 ") the checking flag $F$, whether the sample value of each packet within all observation windows $T_{A_{P Q}}$ is larger than a modified threshold $\mathrm{TH}_{M}$. Then, in the MV-based detection step, the PQD edge detection checkers $C_{f d e t}$ and $C_{b d e t}$ are enabled if the accumulated (summed) flag value "acc $F^{\text {" within }}$ their corresponding windows $T_{f_{P Q}}$ (or $T_{b_{P Q}}$ ) is greater than or equal to $\lceil P / 2\rceil$. That is, in the presented double-edge-based detection procedure, the detection checker $C_{\text {det }}$ becomes "TRUE" only if both $C_{f d e t}$ and $C_{b d e t}$ are enabled. For instance, when assuming $l=3$, if the values of $a c c F$ at the two edge points are greater than or equal to $4(=\lceil P / 2\rceil)$, the PQD event checker is enabled (i.e., $C_{\text {det }}=$ "TRUE"). In addition, if $a c c F$ is greater than or equal to $\lceil P / 2\rceil$ at the outside (or remaining) window $T_{O_{P Q}}$ the false edge detection checker $C_{\text {false }}$ is enabled (i.e., $C_{\text {false }}=$ "TRUE"). 
Below, a list of notations used in this paper and a pseudocode representation of the proposed MV-based detection function (Algorithm 1) are presented, where the modified threshold $T H_{M}$ is used to detect both edges; $l=3$ (or $P=7$ ) is assumed in this algorithm.

As an example, in Figure 5, the proposed MV-based detection algorithm is applied to detect a voltage swell signal with $110 \% p u$ at SNR $=35 \mathrm{~dB}$. Assuming $l=3$, Figure 5 a shows the denoised PQD signal input and Figure $5 b-h$, respectively, shows each of the seven WPCs of that input signal. In this MV-based WPTM metric, the sample values of all WPCs are compared with a threshold value $t h=T H_{M}$ to update the accumulator accF (add one or not (hold)). After accumulating the $2^{l}-1$ WPCs, the PQD event is detected if $a c c F$ is greater than or equal to $\lceil P / 2\rceil$.

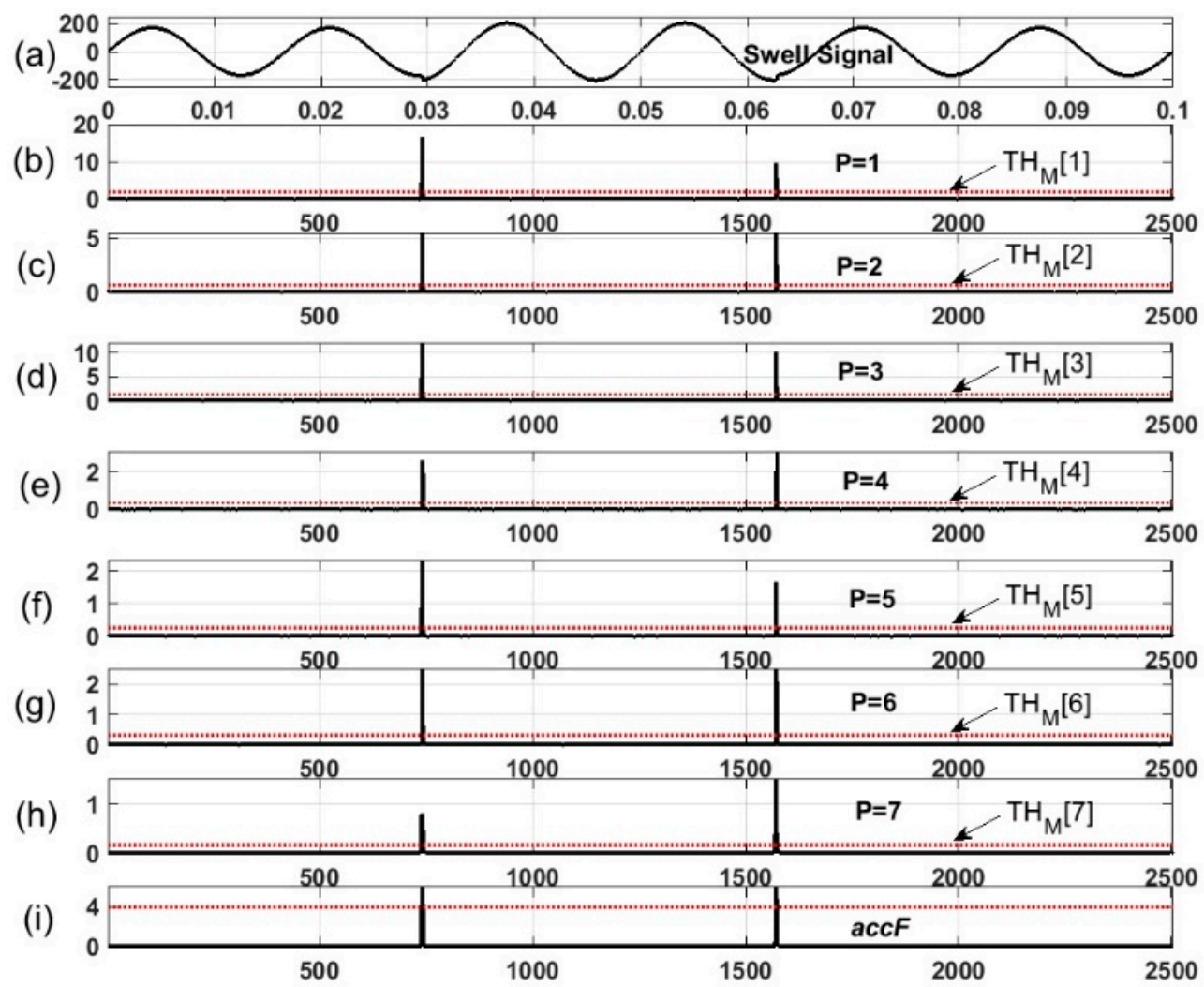

Figure 5. Snapshots of WPTM-based detection using MV: (a) voltage swell signal with $110 \%$ pu, (b) wavelet packet $W^{1}$, (c) $W^{2},(\mathbf{d}) W^{3},(\mathbf{e}) W^{4},(\mathbf{f}) W^{5},(\mathbf{g}) W^{6},(\mathbf{h}) W^{7},(\mathbf{i})$ result of MV detection $(\operatorname{acc} F)$.

\subsubsection{PQD Feature Detection Using RMS Metric}

Next, we define and use the WPTM-based detection scheme with the RMS metric:

$$
\mathbf{M}=\mathbf{M}_{r m s}=\left\{M_{r m s}[n] \mid n=1, \ldots, N\right\},
$$

where,

$$
M_{r m s}[n]=\sqrt{\frac{1}{P} \sum_{p=1}^{P}\left|W^{p}[n]\right|^{2}} .
$$

For RMS-based PQD detection, the proposed algorithm procedure in Figure 1 calls the WPTM-based PQD detection function using the RMS metric (see Algorithm 2; whose pseudocode is shown below), where the following modified threshold is used:

$$
T H_{M}=t h=k \times \operatorname{med}(\boldsymbol{M}) \sqrt{2 \log |N|} / 0.6745 .
$$


While the MV metric uses $P$ measures (one measure per packet), the RMS metric exploits a single combined measure to detect the PQD event. The denoised wavelet packets undergoing the impulsive and/or Gaussian denoising process (Step 2 to Step 4 in Figure 1) are used to obtain the RMS metric, as observed in (19).

In the presented double-edge detection procedure, $C_{\text {det }}$ becomes "TRUE" only if both $C_{f d e t}$ and $C_{b d e t}$ are enabled " 1 ". For instance, if the RMS value $M[t]$ at the two edge points is greater than or equal to the threshold $t h$, then the PQD event is detected (i.e., $C_{d e t}=$ "TRUE"). In addition, if $M[t]$ is greater than or equal to th at the outside (or remaining) window $T_{O_{P Q}}, C_{\text {false }}$ is enabled (i.e., $C_{\text {false }}=$ "TRUE").

As an example, Figure 6a shows snapshots of RMS-based feature detection for a sag signal with $80 \%$ pu at SNR $=40 \mathrm{~dB}$. Assuming $l=3$, Figure $6 \mathrm{~b}-\mathrm{d}$, respectively, shows the first, second, and seventh wavelet packets (or WPCs) (the other packets are omitted for simplicity) of the PQD signal. Figure 6e is the resulting subplot of the WPTM-based RMS metric, that is, $\mathbf{M}=\mathbf{M}_{r m s}$ in (19). A PQD event is detected when the RMS value is larger than the red-dotted threshold line $\left(t h=T H_{M}\right)$.
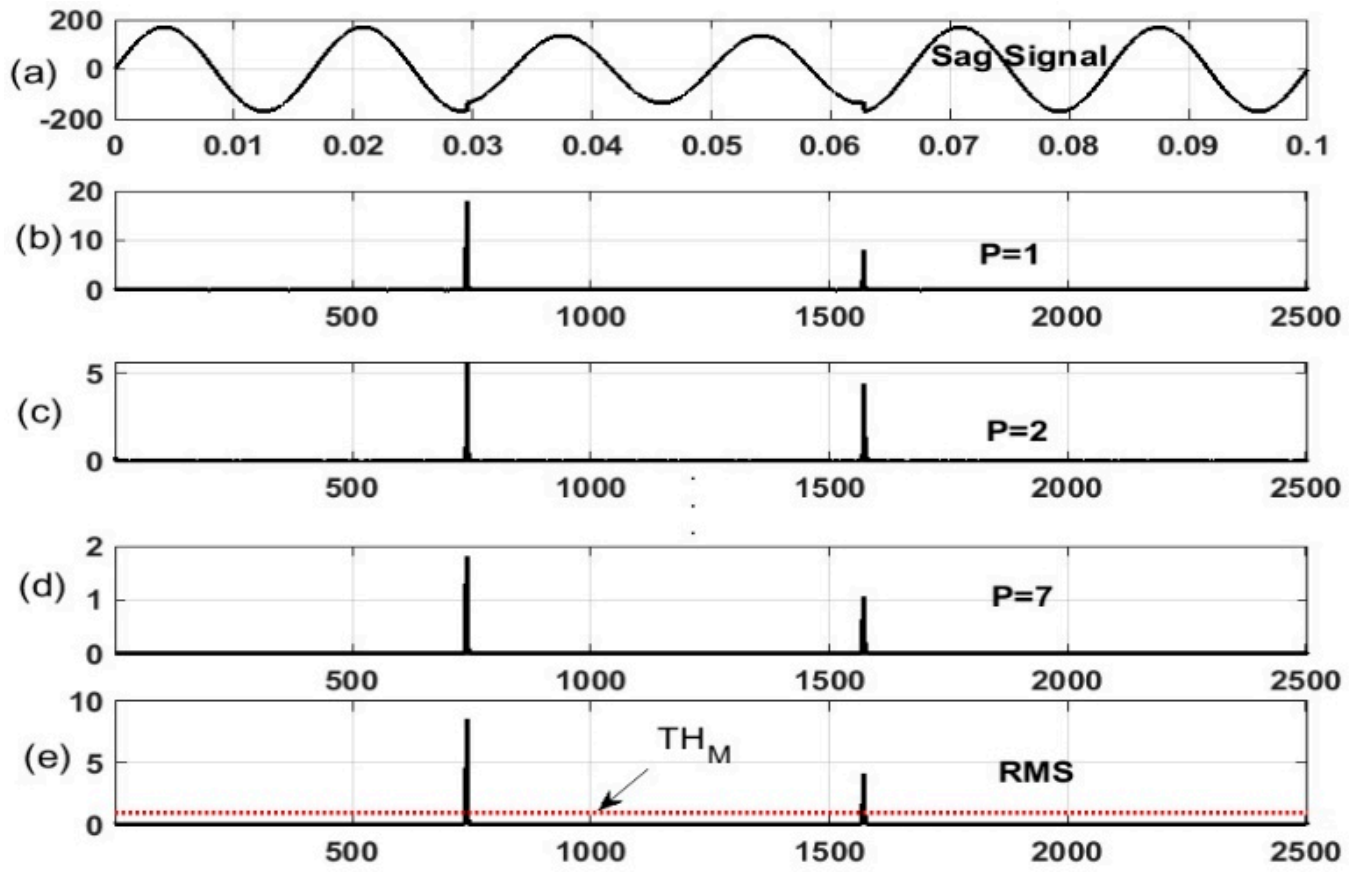

Figure 6. Snapshots of WPTM-based detection using RMS: (a) voltage sag signal with $80 \%$ pu after denoising, (b) wavelet packet $W^{1}$, (c) $W^{2}$, (d) $W^{7},(\mathbf{e})$ result of RMS detection.

Table 3 summarizes the difference between the two metrics (MV and RMS) of the proposed scheme and the wavelet-correlation metric of conventional ("Conv") scheme [1], in terms of complexity, strength, and the $k$ factor values over the simulated different noise channels. While the MV metric compares each $P\left(=2^{l}-1\right)$ wavelet packet with its corresponding threshold th and collects the accumulated result for PQD detection, the RMS metric directly compares a single RMS value derived from $P$ packets with th for PQD detection. Thus, the MV metric is more complex than the RMS metric, but exhibits relatively better DR and worse FER than the RMS metric. On the other hand, the existing scheme [1] using wavelet correlation-based metrics has much higher complexity due to the additional scale adjustment procedure (see Step 4, page 1989 of [1] for the details). In addition, it has lower DR and higher FER than our scheme; their numerical results are compared in detail in the next section. 


\section{Numerical Results and Discussions}

This section presents the numerical results of the proposed WPTM-based PQD detection scheme and compares them with those obtained for the existing wavelet-based denoising (W-Den [10]), WC-Det [1], and non-wavelet-based detection schemes (S-transform [26]). In the simulation, we generate standard PQD event signals [9], including voltage sag, voltage swell, harmonics, sustained interruption, notch, and so on, under real stochastic electricity channel conditions. In the proposed detection scheme, we use RMS or MV metric. Let us assume $l=3$; thus, $P=2^{l}-1=7$ packets are used to detect these PQD events. In the proposed PQD detection procedure, the universal threshold $T H_{U}=\sigma_{d} \sqrt{2 \log |N|}$ is initially used, assuming $N=2504$ (with a sampling rate $f_{s}=1 / T_{s}=2 \times 10^{5}$ samples $/ \mathrm{s}$ ). Then, the proposed algorithm replaces $T H_{U}$ with a modified (or updated) threshold with parameter $k$ (Table 3), $T H_{M}=k \sigma_{d} \sqrt{2 \log |N|}$, depending on the estimated channel and defined metric, such that its robustness is improved under severe stochastic power signal conditions, including impulsive noise, random phase offset, and low SNR. The simulation assumes that the phase offset $\varphi_{m}$ is uniformly distributed, that is, $\varphi_{m} \sim U[0,2 \pi]$, and that impulsive noise is generated following the B-G model [21].

In the following subsections, we numerically validate that the proposed scheme with the adaptive denoising function is superior to the existing schemes over such real statistical channels. In particular, double-edge-based detection, which is chosen for more precise PQD localization, is compared with conventional single-edge-based detection over impulsive and Gaussian noise channels. We then analyze the performance effect of the proposed system model with an adaptive impulsive denoising function in detail and evaluate the proposed model for diverse standard PQDs and generate PQDs from an implemented practical distribution network using MATLAB ${ }^{\circledR}$ Simulink $^{\circledR}$ [27]. Finally, we present a summarized discussion on the numerical analysis of the proposed system model.

\subsection{Effect of Random Phase Offset and Modified Threshold}

PQD feature detection is much sensitive to the start or end edge positions of PQD signals. Hence, the detection scheme must be robust to the random phase offset of the PQD signals, as shown in Figure 4. For this purpose, the proposed scheme uses a modified threshold $T H_{M}$ rather than a universal threshold $T H_{U}$, which results in a lower FER $(<10 \%$ for Gaussian noise) while maintaining suitable DR, even though it is still low at $\varphi_{m} \approx \pi / 2$ or $3 \pi / 2$ (Figure 4 ).

Table 2 compares the performance (in terms of DR and FER) of the proposed scheme (herein, assuming double edge detection) for a sag signal of $80 \% \mathrm{pu}$ with a fixed phase offset (assuming $\varphi_{m}=\pi$, i.e., "fixed PQD") or random phase offset (assuming $\varphi_{m} \sim U[0,2 \pi$ ), i.e., "random $\mathrm{PQD}$ "). In this simulation, $\mathrm{MV}$ is used as the detection metric and a Gaussian noise channel with SNR $=40 \mathrm{~dB}$ is assumed. Table 2 shows that for both fixed and random PQDs, the presented MV metric with $T_{M}$ dominates the same metric with $T H_{U}$ [7] in terms of FER. Hereinafter, unless stated otherwise, $T H_{M}$ with $k$ factor (Table 3 ) is used for the proposed scheme.

\subsection{Single- and Double-Edge-Based Detection}

We evaluate the performance of the proposed scheme in terms of both double- and single-edge-based detection. In Table 4, the single-edge-based detection performance of both the proposed algorithm (denoted as "Prop") and conventional WC-Det algorithm ("Conv") is compared over either Gaussian noise channels (AWGN) or impulsive noise channels (IMPN). 
Table 4. Single-edge-based PQD detection performance (DR/FER (\%)) of the proposed and conventional wavelet-correlation-based-detection (WC-Det) algorithms, where a swell signal with $120 \%$ $p u$ is used over Gaussian noise (AWGN) with signal-to-noise ratio (SNR) $=35 \mathrm{~dB}$ or IMPN with $\mathrm{SNR}=35 \mathrm{~dB}, \mu=400, \psi=0.02 \%$.

\begin{tabular}{cccccc}
\hline & & \multicolumn{2}{c}{ Fixed PQD } & \multicolumn{2}{c}{ Random PQD } \\
\cline { 3 - 6 } & & AWGN & IMPN & AWGN & IMPN \\
\hline \multirow{2}{*}{ Prop. } & MV & $100 / 0$ & $100 / 0.1$ & $89.8 / 8.6$ & $89.2 / 11.6$ \\
& RMS & $100 / 0$ & $100 / 0.1$ & $89.6 / 6.4$ & $88.8 / 11.6$ \\
\hline Conv. & WC-Det. & $100 / 0$ & $100 / 8.3$ & $80.1 / 11$ & $74.6 / 58.2$ \\
\hline
\end{tabular}

As the simulated PQD event, a swell signal with $120 \% p u$ and $\varphi_{m}=\pi$ (for fixed PQD) or $\varphi_{m} \sim U[0,2 \pi$ ) (for random PQD) is used. As indicated in Table 4, the proposed scheme has approximately $9-10 \%$ higher DR and 2-5\% lower FER for random PQD over AWGN, as compared to WC-Det. In contrast, the proposed scheme has approximately $14-15 \%$ higher DR and approximately $47 \%$ lower FER for random PQD over IMPN, as compared to WC-Det. This indicates that the proposed scheme has an improved FER and DR compared to WC-Det, especially for random PQD. For fixed PQD, the proposed scheme is slightly better ( $8 \%$ lower over IMPN) in terms of FER than WC-Det while maintaining the same DR.

Comparing MV and RMS, especially for random PQD, while MV is relatively better than RMS in terms of DR, RMS is relatively better than MV in terms of FER. While assuming single-edge-based PQD detection, Table 4 shows that the proposed (MV or RMS) scheme has a similar DR for fixed PQD but a slightly improved DR for random PQD, as compared to the existing scheme (WC-Det). In terms of FER (for both fixed PQD and random PQD), the proposed scheme is superior to the existing scheme, especially over IMPN.

In most previous studies, including Liao's papers [1,28], only single-edge-based PQD detection has been used. However, for precise PQD localization, double edge-based detection identifying both (start and end) edges of a PQD signal is necessary. In the simulation, we set the allowable limit of error (ALE) time of double-edge-based detection to be within $\pm 7.5 \times 10^{-5} \mathrm{~s}$ ( \pm 15 samples). This means that the PQD detection position (time) can deviate as much as the ALE time from the actual position of PQD occurrence. The accuracy of PQD detection position (time) decreases as the ALE time increases. Table 5 compares the DR of double-edge-based PQD over AWGN or IMPN channel, where SNR $=35 \mathrm{~dB}$ or 40 $\mathrm{dB}$ is assumed, between the proposed and existing schemes (WC-Det and W-Den). For the simulated PQD, we generate either (a) a sag signal with $80 \%$ pu or (b) a swell signal with $120 \% p u$. Table 5 shows that, in case of fixed PQD, there is no large difference between the existing and proposed schemes in terms of DR. However, in case of random PQD, the proposed scheme has much more improved DR over AWGN or IMPN than the existing wavelet-based schemes (WC-Det and W-Den). Specifically, for signal (a) over AWGN with SNR $=35 \mathrm{~dB}$ and $40 \mathrm{~dB}$, the proposed MV/RMS exhibits 5.9\%/5.4\% and 8.8\%/8.7\% (or $34.5 \% / 34 \%$ and $16.8 \% / 16.7 \%$ ) better DR, respectively, than WC-Det (or W-Den). For the signal (b) over AWGN with SNR $=35 \mathrm{~dB}$ and $40 \mathrm{~dB}$, the proposed MV/RMS exhibits $7.7 \% / 6.9 \%$ and $9.1 \% / 8.9 \%$ (or $34.6 \% / 33.8 \%$ and $23.6 \% / 23.4 \%$ ) better DR, respectively, than WC-Det (or W-Den).

When considering random PQD over IMPN, the proposed scheme is more dominant than the conventional wavelet-based schemes. For instance, for the signal (a) over IMPN with SNR $=35 \mathrm{~dB}$ and $40 \mathrm{~dB}$, the proposed MV/RMS exhibits $26.9 \% / 26.4 \%$ and $13.5 \% / 13.2 \%$ (or $48.9 \% / 48.4 \%$ and $30.9 \% / 30.6 \%$ ) better DR, respectively, than WC-Det (or W-Den). For the signal (b) over IMPN with SNR $=35 \mathrm{~dB}$ and $40 \mathrm{~dB}$, the proposed MV/RMS exhibits $27.4 \% / 25.4 \%$ and $13.4 \% / 13 \%$ (or $52.6 \% / 50.6 \%$ and $32.2 \% / 31.8 \%$ ) better DR, respectively, than WC-Det (or W-Den). In Table 5, the proposed scheme is also compared with the S-transform-based scheme $[3,27]$ as a non-wavelet-based detection. We confirm that the S-transform exhibits much lower DR (even worse than those of WC-Det and W-Den), especially in the case of random PQD, as compared to the proposed scheme. 
Table 5. Double-edge-based PQD detection performance (DR (\%)) of the proposed and conventional (WC-Det, W-Den, and S-Transform) algorithms, where (a) a sag signal with $80 \%$ pu or (b) SWELL: a swell signal with $120 \%$ pu is used over AWGN or IMPN with $\mathrm{SNR}=35 \mathrm{~dB}$ or $40 \mathrm{~dB}$.

\begin{tabular}{|c|c|c|c|c|c|c|c|c|c|c|c|c|c|}
\hline & & \multicolumn{4}{|c|}{$\begin{array}{c}\text { Fixed PQD } \\
(\mathrm{SNR}=35 \mathrm{~dB})\end{array}$} & \multicolumn{4}{|c|}{$\begin{array}{l}\text { Random PQD } \\
(\mathrm{SNR}=35 \mathrm{~dB})\end{array}$} & \multicolumn{4}{|c|}{$\begin{array}{l}\text { Random PQD } \\
(\mathrm{SNR}=40 \mathrm{~dB})\end{array}$} \\
\hline & & \multicolumn{2}{|c|}{ AWGN } & \multicolumn{2}{|c|}{ IMPN } & \multicolumn{2}{|c|}{ AWGN } & \multicolumn{2}{|c|}{ IMPN } & \multicolumn{2}{|c|}{ AWGN } & \multicolumn{2}{|c|}{ IMPN } \\
\hline & & (a) & (b) & (a) & (b) & (a) & (b) & (a) & (b) & (a) & (b) & (a) & (b) \\
\hline \multirow{2}{*}{ Prop. } & MV & 100 & 100 & 100 & 100 & 82.3 & 78.5 & 86.3 & 84.0 & 89.0 & 87.9 & 90.1 & 89.4 \\
\hline & RMS & 100 & 100 & 100 & 100 & 81.8 & 77.7 & 85.8 & 82.0 & 88.9 & 87.7 & 89.8 & 89.0 \\
\hline \multirow{3}{*}{ Conv. } & WC-Det. & 100 & 100 & 98.8 & 93.0 & 76.4 & 70.8 & 59.4 & 56.6 & 80.2 & 78.8 & 76.6 & 76.0 \\
\hline & W-Den. & 100 & 99.5 & 98 & 86.8 & 47.8 & 43.9 & 37.4 & 31.4 & 72.2 & 64.3 & 59.2 & 57.2 \\
\hline & S-Tran. & 100 & 100 & 100 & 100 & 18.7 & 22.3 & 22.0 & 21.5 & 21.8 & 18.5 & 17.5 & 28.0 \\
\hline
\end{tabular}

A comparison of Tables 4 and 5 indicates that the proposed scheme is superior to the existing schemes in terms of both single- and double-edge-based detection performance. Particularly, for the double-edge-based detection of random PQD (Table 5), the proposed MV or RMS scheme has much more improved performance indices (i.e., in terms of DR and FER; even FER results are omitted in Table 5 for simplicity) than the existing schemes. A comparison between the proposed metrics indicates that MV is slightly better than RMS, especially in terms of double-edge-based DR.

\subsection{Effect of Adaptive Impulsive Denoising}

In Table 6, we assume that a sag signal of $80 \% p u$ under the randomly phased IMPN conditions with SNR $=20$ to $40 \mathrm{~dB}, \mu=400$, and $\psi=0.02 \%$ is generated. The results in Table 6 show that the proposed scheme with the adaptive denoising function (see Step 4 in Figure 1) effectively eliminates impulsive noise. In addition, the proposed algorithm considerably reduces the FER under severe impulsive electricity channel conditions, even at a very low SNR $(\leq 25 \mathrm{~dB})$. Moreover, the proposed scheme with adaptive impulsive denoising (case (b) in Table 6) exhibits more improved FER and DR than the same scheme without adaptive impulsive denoising (i.e., only with wavelet-based Gaussian denoising; case (a) in Table 6). Specifically, by adding the impulsive denoising function, the proposed MV and RMS methods exhibit an improved DR of $7.5 \%$ and $8.6 \%$ (or $11.3 \%$ and $13.7 \%$ ) at SNR $=35 \mathrm{~dB}$ (or SNR $=25 \mathrm{~dB}$ ), respectively, while considerably reducing the FER. At $\mathrm{SNR}=40 \mathrm{~dB}$, the proposed algorithm with the impulsive denoising step (Step 4) exhibits a slightly better $(<1.3 \%)$ DR while maintaining a suitable FER. As a result, we confirm that adaptive impulsive denoising makes the proposed detection algorithm more robust to a real IMPN environment.

Table 6. Performance comparison (DR/FER (\%)) between (a) random PQD detection without impulsive denoising and (b) random PQD detection with impulsive denoising, where an $80 \%$ pu sag signal over IMPN with SNR $=20,25,35$, or $40 \mathrm{~dB} ; \mu=400$; and $\psi=0.02 \%$ is assumed.

\begin{tabular}{cccc}
\hline SNR & Metric & (a) w/o Impulsive Denoising & (b) w/Impulsive Denoising \\
\hline \multirow{2}{*}{$40 \mathrm{~dB}$} & MV & $89.6 / 89.9$ & $90.1 / 11.7$ \\
& RMS & $88.5 / 89.6$ & $89.8 / 7.5$ \\
\hline \multirow{2}{*}{$35 \mathrm{~dB}$} & MV & $78.8 / 94.5$ & $86.3 / 9.2$ \\
& RMS & $77.2 / 94.1$ & $85.8 / 8.0$ \\
\hline \multirow{2}{*}{$25 \mathrm{~dB}$} & MV & $49.2 / 88.7$ & $60.5 / 14.0$ \\
& RMS & $45.3 / 88.2$ & $59.0 / 15.1$ \\
\hline \multirow{2}{*}{$20 \mathrm{~dB}$} & MV & $13.8 / 90.8$ & $34.5 / 14.9$ \\
& RMS & $10.4 / 90.3$ & $32.7 / 17.3$ \\
\hline
\end{tabular}




\subsection{Detection of Diverse PQD Types}

Within a power grid, various PQD events can occur. Herein, we focus on six major PQD events-sag, swell, interruption, harmonics, notch, and oscillatory transient-and two combined PQD events-swell with harmonics and sag with harmonics, which can often be generated within the power grid and cause serious problems in the connected electric devices or facilities.

Table 7 summarizes the numerical results of the proposed detection scheme for the diverse randomly phased PQD events $\left(\varphi_{m} \sim U[0,2 \pi)\right.$; that is, random PQDs) that are generated over the IMPN channels with SNR $=40 \mathrm{~dB}, \mu=400$, and $\psi=0.02 \%$. In the simulation, the critical PQD events specified by IEEE1159 (Table 1) (e.g., a swell with 110\% $p u$, a sag with $90 \% p u$, and harmonics ("Har") with $10 \% p u$ ) are assumed to occur. In this simulation, we assume that such PQD event signals have a limited duration of two cycles (which are specified as "instantaneous"- or "momentary"-type signals by IEEE1159).

Table 7. Detection performance (DR (\%)) of double-edge-based random PQD events using the proposed algorithm, where a sag signal with $90 \% p u$, a swell signal with $110 \% p u$, a harmonics signal with $10 \% p u$, interruption, notch, oscillatory transient signal, and swell (or sag) with harmonics are assumed over IMPN channels of SNR $=40 \mathrm{~dB}, \mu=400$, and $\psi=0.02 \%$

\begin{tabular}{ccc}
\hline PQD Events & MV & RMS \\
\hline Swell 110\% & 78.5 & 78.2 \\
Sag $90 \%$ & 81.1 & 81.0 \\
Har. 10\% & 100 & 100 \\
Int & 100 & 100 \\
Notch & 100 & 100 \\
Oscillatory transient & 100 & 100 \\
Swell 110\% with Har. 10\% & 100 & 100 \\
Sag 110\% with Har. 10\% & 100 & 100 \\
\hline
\end{tabular}

Table 7 shows that a swell with $1.1 \mathrm{pu}$ (or a sag with $0.9 \mathrm{pu}$ ) exhibits much more degraded DR than that with $1.2 \mathrm{pu}$ (or a sag with $0.8 \mathrm{pu}$; Table 5). This indicates that the amplitude of the PQD signals substantially affects the detection performance. In addition, a comparison of MV and RMS indicates that MV is only slightly better in terms of DR than RMS.

The table also shows that the proposed scheme exhibits almost 100\% DR for harmonics, interruption, notch, oscillatory transient, and swell (or sag) with harmonics [29], which indicates that these PQD events are relatively easy to detect as compared to pure sag or swell.

\subsection{Simulation for a Distribution Network}

We implement a simple power distribution network with line faults using MATLAB ${ }^{\circledR}$ Simulink ${ }^{\circledR}$, as shown in Figure $7 \mathrm{a}$, which generates PQD event signals such as voltage sag and voltage swell, and we test the proposed system model for the created event signals. We also implement a nonlinear load network using MATLAB ${ }^{\circledR}$ Simulink ${ }^{\circledR}$, as shown in Figure $7 \mathrm{~b}$, which generates harmonic voltage disturbance signals. Those models comprise $11 \mathrm{KV}, 30 \mathrm{MVA}$, and $60 \mathrm{~Hz}$ three-phase source block feeding through $0.4 \mathrm{KV}, 1 \mathrm{MVA}$ delta/wye transformers to a $10 \mathrm{KW}$ resistive and 100 VAR inductive load (whose detailed properties can be referred to from [27]). In a practical network model [27], we can insert line faults generating voltage sage or swell events. The PQD events generated by this network are assumed to have a fixed phase (i.e., fixed PQD). 


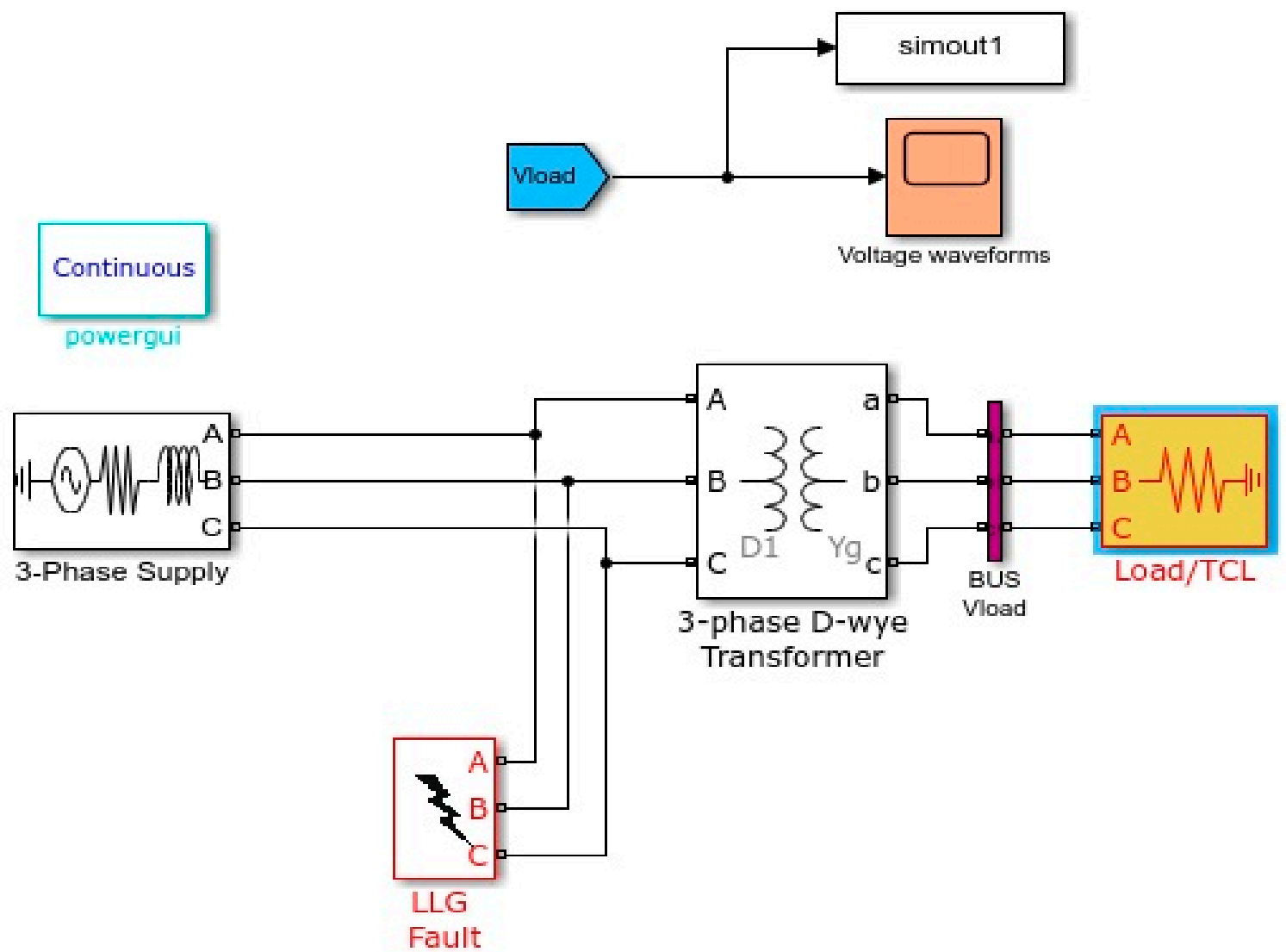

(a)

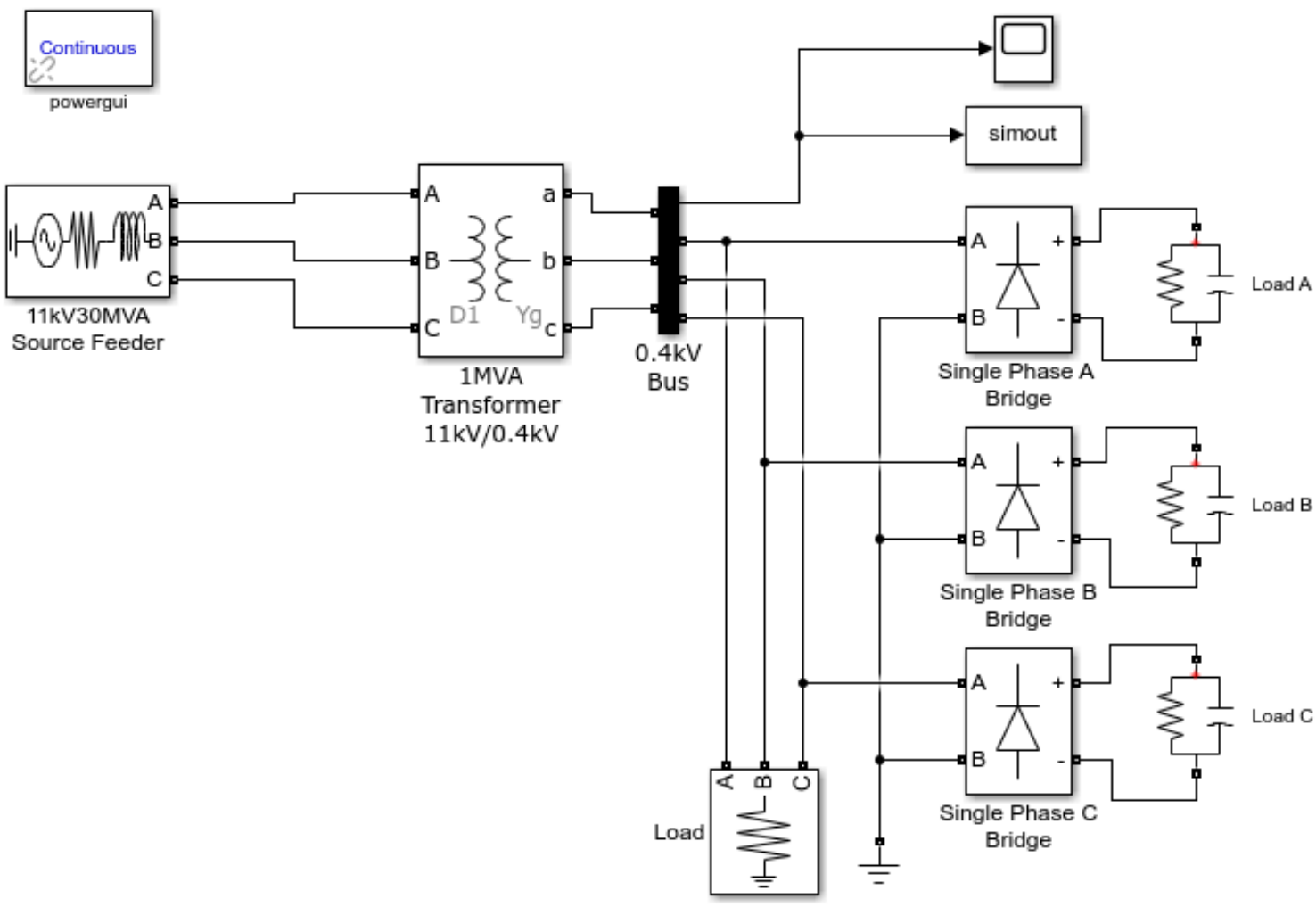

(b)

Figure 7. (a) Implemented network model with line faults using Simulink ${ }^{\circledR}$, (b) Nonlinear load model using Simulink ${ }^{\circledR}$. 
Figure 8 shows the voltage sag (Phases A and B) and voltage swell (Phase C) waveforms generated by the corresponding line faults of the power distribution network in Figure 7a. A simulation time of $0.2 \mathrm{~s}$ is set and the ode23tb solver is selected to run the simulation. From the above waveforms shown in Figure 8, we choose Phase B for the voltage sag signal with $78 \% p u$ and Phase $C$ for the voltage swell signal with $111 \% p u$, and then apply our proposed algorithm to the PQD event signals generated over AWGN channels with SNR $=30-35 \mathrm{~dB}$ and IMPN channels with SNR $=30-35 \mathrm{~dB}, \mu=400$, and $\psi=0.02 \%$, respectively, to evaluate the detection performance.

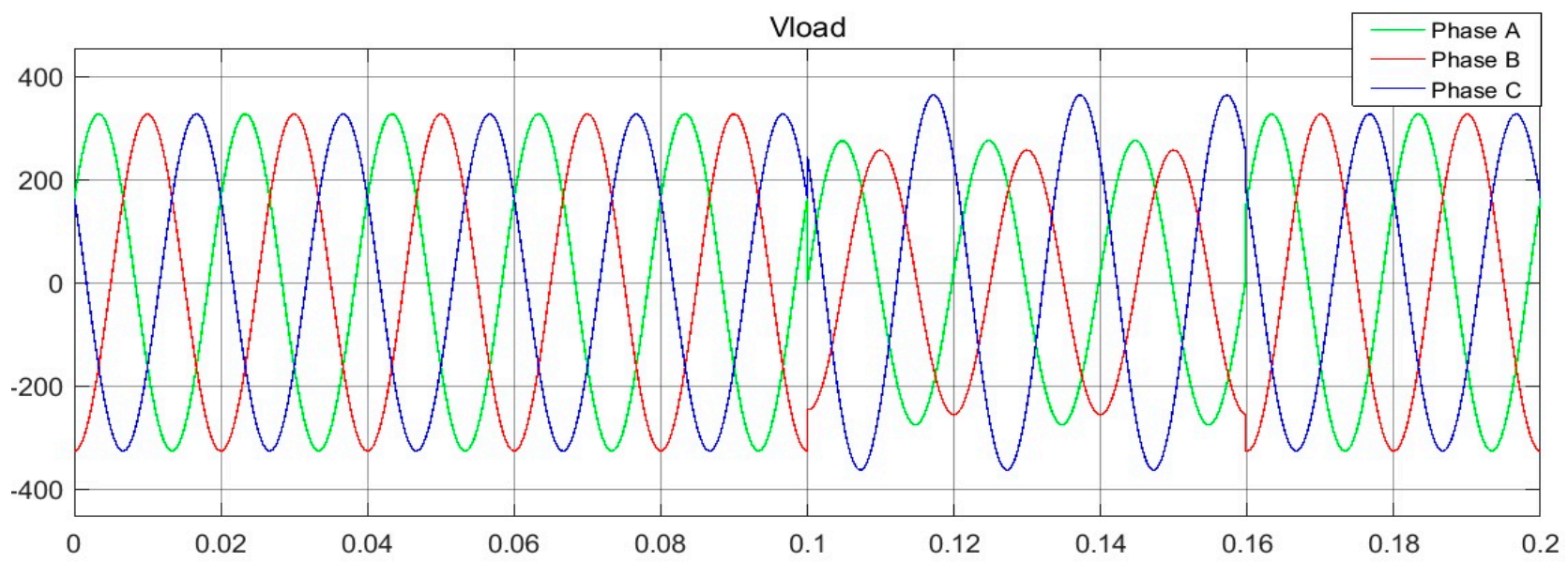

Figure 8. Voltage sag (Phases A and B) and swell (Phase C) waveforms.

Table 8 shows the performance of the double-edge-based PQD detection system over AWGN channels with SNR $=30-35 \mathrm{~dB}$ and IMPN channels with SNR $=30-35 \mathrm{~dB}, \mu=400$, and $\psi=0.02 \%$.

Table 8. Double-edge-based detection performance (DR/FER (\%)) of the proposed algorithm, for which a sag signal with $78 \% p u$, a swell signal with $111 \% p u$ generated from the implemented network model of Figure 7a, and a harmonics signal with $10 \%$ pu generated from the implemented network model of Figure $7 \mathrm{~b}$ are generated over AWGN channels with SNR $=30-35 \mathrm{~dB}$ or IMPN channels with $\mathrm{SNR}=30-35 \mathrm{~dB}, \mu=400$, and $\psi=0.02 \%$.

\begin{tabular}{cccccccc}
\hline & \multicolumn{7}{c}{ Fixed PQD } \\
\hline \multirow{2}{*}{ SNR } & & \multicolumn{7}{c}{ AWGN } & & & IMPN \\
& & Sag & Swell & Har. & Sag & Swell & Har. \\
\cline { 3 - 8 } & MV & $100 / 5.8$ & $100 / 3.0$ & $100 / 1.9$ & $100 / 0.0$ & $100 / 0.0$ & $100 / 0.0$ \\
\multirow{3}{*}{$35 \mathrm{~dB}$} & RMS & $100 / 3.8$ & $100 / 2.8$ & $100 / 1.8$ & $100 / 0.0$ & $100 / 0.0$ & $100 / 0.0$ \\
& WC-Det. & $100 / 20.6$ & $100 / 25.2$ & $57.6 / 52.7$ & $100 / 99.6$ & $100 / 99.8$ & $42.4 / 94.0$ \\
\hline \multirow{3}{*}{$30 \mathrm{~dB}$} & MV & $100 / 6.4$ & $100 / 5.6$ & $99.5 / 1.8$ & $100 / 0.0$ & $100 / 0.0$ & $100 / 0.0+$ \\
& RMS & $100 / 5.8$ & $100 / 5.0$ & $100 / 1.2$ & $100 / 0.4$ & $100 / 0.0$ & $100 / 0.0$ \\
& WC-Det. & $100 / 11.4$ & $100 / 15.4$ & $53.8 / 54.6$ & $99.6 / 100$ & $96.2 / 100$ & $40.6 / 94.4$ \\
\hline
\end{tabular}

The results of the network distribution model (Figure 7a) and the nonlinear load model (Figure $7 \mathrm{~b}$ ) over AWGN or IMPN channel, as observed in Table 8, verify that the proposed system model is suitable for real grid systems. Compared to the proposed schemes (MV or RMS), the conventional scheme (WC-Det) has a similar DR for sag or swell signals but has a degraded DR for harmonic signals over AWGN or IMPN channels. However, WC-Det has a higher FER than the proposed schemes, especially for PQD over IMPN. Table 8 also shows that both MV and RMS have comparable performance over AWGN and IMPN channels. 


\subsection{Discussion}

Most of the existing PQD detection schemes have been evaluated over generic electricity channels using only Gaussian noise (AWGN). Hence, the PQD detection analysis under practically severe SG channel conditions, including random phase offset, impulsive noise, and low SNR $(\leq 40 \mathrm{~dB})$, has been limited. For instance, a numerical comparison between fixed PQD and random PQD in Tables 4 and 5 indicates that the PQD detection performance is considerably dependent upon the random phase offset. The impulsive noise, which is mainly due to the power switch ON/OFF or man-made EMI, can also substantially affect the performance of PQD detection or localization [2] (Tables 4-8).

Throughout this section, we analyze the proposed WPTM-based PQD detection system model numerically under such severe electricity channel conditions and compare it with the existing system models. Specifically, in case of PQD events with a fixed phase offset (see fixed PQD in Tables 3-5), there is no significant difference in DR between the proposed and conventional methods (there is even some more difference of FER over IMPN channels). However, in case of PQD events with random phase offset (see random PQD in Tables 4 and 5), the proposed method using WPTM-based metrics (MV and RMS) is relatively superior to conventional wavelet-based (WC-Det and W-Den) or non-wavelet-based (S-Tran) methods, especially over IMPN.

When considering the precise detection or localization of PQD events with a limited duration, double-edge-based detection is more favorable than conventional single-edgebased detection. In particular, for random PQD (i.e., PQD event signal with a uniformly distributed random phase: $\varphi_{m} \sim U[0,2 \pi)$ ) in Table 5, the simulation results obtained for AWGN or IMPN indicate that the proposed scheme using MV or RMS metric is much superior to the existing wavelet-based or non-wavelet-based scheme in terms of doubleedge-based detection performance (DR or FER). Especially for the IMPN channel with a very low $\mathrm{SNR}(\leq 25 \mathrm{~dB})$, the DR difference between the proposed and existing schemes increases further.

The simulation results in Tables 6 and 7 indicate that the proposed PQD detection algorithm (see Figure 1 in Section 2), which has an adaptive impulsive denoising function, improves the robustness under the IMPN channel conditions. Specifically, Table 6 shows that the adaptive impulsive denoising step effectively eliminates impulsive noise; thus, the proposed MV or RMS significantly reduces FER while maintaining a suitable DR. Table 7 also shows that the presented algorithm works well for various PQD events, including harmonics, interruption, notch, oscillatory transient, and swell (or sag) with harmonics, as well as pure sag or swell. According to the simulation results, MV is superior to RMS in terms of DR, whereas RMS is superior to MV in terms of FER.

Through the simulated experiment of real network distribution models (Table 8), we verify that the proposed MV or RMS scheme guarantees better DR and FER over both AWGN and IMPN channels, as compared to the existing scheme (WC-Det). In particular, the performance difference between the proposed and existing schemes further increases as the SNR decreases (Tables 5 and 8).

The results of the numerical analysis indicate that the proposed PQD detection scheme with WPTM-based metrics is more reliable and has better detection capability under severe random electricity channel conditions than the conventional schemes.

\section{Conclusions}

This paper presents a WPTM-based PQD detection algorithm that is applicable to reliable smart grid construction and demonstrates its simulation under real stochastic electricity channel conditions, including random phase offset, impulsive noise, and low SNR. We define several WPTM-based metrics, such as MV and RMS, that exhibit more degrees of freedom than conventional WTM-based metrics. In addition, in the proposed PQD feature detection scheme, we define a modified threshold $\left(\mathrm{TH}_{\mathrm{M}}\right)$ rather than a conventional universal threshold $\left(\mathrm{TH}_{U}\right)$, which makes it more robust against such severe electricity channel conditions. The simulation results indicate that the proposed algorithm, 
which has an SM filter-based adaptive denoising function, is robust to such severe channel environments, including impulsive noise. The results of the numerical analysis indicate that the proposed scheme is more reliable and has a better detection capability than the existing wavelet-based or wavelet packet-based and non-wavelet-based detection schemes. Furthermore, the presented algorithm is found to work well for various PQD events. Using the experiment for an implemented distribution network with MATLAB ${ }^{\circledR}$ Simulink ${ }^{\circledR}$, we prove that the proposed MV or RMS scheme guarantees better performance over both AWGN and IMPN channels.

Author Contributions: Conceptualization, J.Y. and S.C.; methodology, S.C.; software, J.Y.; validation, J.Y.; formal analysis, J.Y. and S.C.; investigation, J.Y. and S.C.; data curation, J.Y.; writing-original draft preparation, J.Y.; writing-review and editing, S.C.; visualization, J.Y.; supervision, S.C.; project administration, S.C.; funding acquisition, S.C. All authors have read and agreed to the published version of the manuscript.

Funding: This work was funded by the Korean government (MSIT) (2017R1A2B4005840) and the Catholic University of Korea, Research Fund, 2021.

Institutional Review Board Statement: Not applicable.

Informed Consent Statement: Not applicable.

Data Availability Statement: No new data were created or analyzed in this study. Data sharing is not applicable to this article.

Conflicts of Interest: The authors declare no conflict of interest.

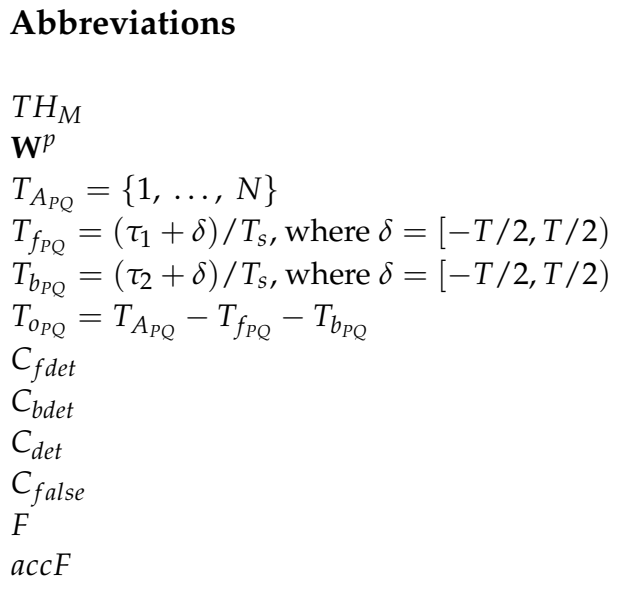

\author{
modified threshold \\ $p$ th wavelet packet \\ entire observation window \\ front edge window of the PQD event \\ back edge window of the PQD event \\ remaining window \\ front edge detection checker \\ back edge detection checker \\ detection checker \\ false edge detection checker \\ edge detection flag \\ edge detection flag accumulator
}

\section{References}

1. Liao, C.-C.; Yang, H.-T.; Chang, H.-H. Denoising Techniques with a Spatial Noise-Suppression Method for Wavelet-Based Power Quality Monitoring. IEEE Trans. Instrum. Meas. 2011, 60, 1986-1996. [CrossRef]

2. Mallat, S. A Wavelet Tour of Signal Processing the Sparse Way; Elsevier: Amsterdam, The Netherlands, 2008; Volume 3, pp. 565-566.

3. Ali, E.; Parisa, E. A new S-transform-based method for identification of power quality disturbances. Arab. J. Sci. Eng. 2017, 43, 2817-2832.

4. $\mathrm{Gu}, \mathrm{J}$. Wavelet threshold de-noising of power quality signals. In Proceedings of the 5th International Conference Natural Computation, Tianjin, China, 14-16 August 2009; pp. 591-597.

5. Li, G.; Zhou, M.; Luo, Y.; Ni, Y. Power quality disturbance detection based on mathematical morphology and fractal technique. In Proceedings of the 2005 IEEE/PES Transmission and Distribution Conference and Exhibition: Asia and Pacific, Dalian, China, 15-18 August 2005; pp. 1-6.

6. Shukla, S.; Mishra, S.; Singh, B. Power quality event classification under noisy conditions using EMD-based de-noising techniques. IEEE Trans. Ind. Inf. 2014, 10, 1044-1054. [CrossRef]

7. Bhuiyan, S.M.A.; Khan, J.; Murphy, G. WPD for detecting disturbances in presence of noise in smart grid for PQ monitoring. IEEE Trans. Ind. Appl. 2018, 54, 702-711. [CrossRef]

8. Selesnick, I.W.; Baraniuk, R.G.; Kingsbury, N.G. The dual-tree complex wavelet transform. IEEE Signal Process. Mag. 2005, 22, 123-151. [CrossRef]

9. IEEE Working Group P1159. Recommended Practice Monitoring Electric Power Quality-Draft 7; IEEE: New York, NY, USA, 1994. 
10. Emanuel, F.; Beti, T.; Sarah, O.; Herwig, R. Comparison of wavelet and Fourier analysis in power quality. In Proceedings of the 2012 Electric Power Quality and Supply Reliability, Tartu, Estonia, 12-16 June 2012.

11. Sifuzzaman, M.; Islam, M.R.; Ali, M.Z. Application of wavelet transform and its advantages compared to Fourier transform. J. Phys. Sci. 2009, 13, 121-134.

12. Swarnabala, U.; Sanjeeb, M. Power quality disturbance detection using wavelet based signal processing. In Proceedings of the 2013 Annual IEEE India Conference, Mumbai, India, 15-28 December 2013.

13. Fathi, A.; Naghsh-Nilchi, A.R. Efficient image denoising method based on a new adaptive wavelet packet thresholding functions. IEEE Trans. Image Process. 2012, 21, 3981-3990. [CrossRef] [PubMed]

14. Hyydt, G.T.; Fjeld, P.S.; Liu, C.C.; Pierce, D.; Tu, L.; Hensley, G. Application of the windowed FFT to electric PQ assessment. IEEE Trans. Power Deliv. 1999, 14, 1411-1416. [CrossRef]

15. Cataliotti, A.; Cosentino, V.; Nuccio, S. A phase-locked loop for the synchronization of power quality instruments in the presence of stationary and transient disturbances. IEEE Trans. Instrum. Meas. 2007, 56, 2232-2239. [CrossRef]

16. Huang, Y.; Liu, Y.; Hong, Z. Detection and location of power quality disturbances based on mathematical morphology and Hilbert-Huang transform. In Proceedings of the 9th International Conference Electronic Measurement \& Instruments, Beijing, China, 23-25 August 2009; pp. 319-324.

17. Manikandan, M.S.; Soman, K.P. A novel method for detection R-peaks in electrocardiogram (ECG) signal. Biomed. Signal Process. Control 2012, 7, 118-128. [CrossRef]

18. Saurabh, K.; Ishita, D. Detection of power quality disturbances using wavelet transform and artificial neural network. In Proceedings of the 2014 AICERA/iCMMD, Kottayam, India, 16-18 July 2014.

19. Golroudbari, M.A. Signal denoising based on wavelet transform using a multi-level threshold function. In Proceedings of the 7th International Conference Application of Information and Communication Technologies, Baku, Azerbaijan, 16-23 October 2013; pp. 1-5.

20. Yoo, J.; Choe, S. Detector of power quality disturbance signal with random phase offset. IEICE Electron. Express 2019, 16, 1-4. [CrossRef]

21. Do, G.N.; Siohan, P.; Helene, M. Adaptive noise mitigation in impulsive environment: Application to power-line communications. IEEE Trans. Power Deliv. 2010, 25, 647-656.

22. Juneja, M.; Sandhu, P.S. Design and development of an improved adaptive median filtering method for impulse noise detection. Int. J. Comput. Electr. Eng. 2009, 1, 627-630. [CrossRef]

23. Coifman, R.R.; Wickerhauser, M.V. Entropy-based algorithms for best basis selection. IEEE Trans. Inf. Theory 1992, 38, 713-718. [CrossRef]

24. Donoho, D.L. De-noising by soft thresholding. IEEE Trans. Inf. Theory 1995, 41, 613-627. [CrossRef]

25. Perunicic, B.; Mallini, M.; Wang, Z.; Liu, Y. Power quality disturbance detection and classification using wavelets and artificial neural networks. In Proceedings of the IEEE 8th International Conference on Harmonics and Quality of Power, Athens, Greece, 14-16 October 1998; pp. 77-82.

26. Huda, N.H.T.; Abdullah, A.R.; Jopri, M.H. Power quality signals detection using S-transform. In Proceedings of the 2013 IEEE 7th International Power Engineering and Optimization Conference (PEOCO), Langkawi, Malaysia, 3-4 June 2013.

27. Rodney, H.G.T.; Vigna, K.R. A comprehensive modeling and simulation of power quality disturbances using MATLAB/SIMULINK. In Power Quality Issues in Distributed Generation; Intech: Rijeka, Croatia, 2015; pp. 83-107.

28. Liao, C.-C. Enhanced RBF Network for Recognizing Noise-Riding Power Quality Events. IEEE Trans. Instrum. Meas. 2010, 59, 1550-1561. [CrossRef]

29. Kumar, R.; Singh, B.; Shahani, D.T. Recognition of Single-stage and Multiple Power Quality Events Using Hilbert-Huang Transform and Probabilistic Neural Network. Electr. Power Compon. Syst. 2015, 43, 607-619. [CrossRef] 